\title{
A real-life comparative effectiveness study into the addition of antibiotics to the management of asthma exacerbations in primary care
}

Clare S Murray ${ }^{1}$, Sarah J Lucas ${ }^{2}$, John Blakey ${ }^{3}$, Alan Kaplan ${ }^{4}$, Alberto Papi ${ }^{5}$, James Paton ${ }^{6}$, Wanda

Phipatanakul ${ }^{7}$, David Price ${ }^{8}$, Oon Hoe Teoh ${ }^{9}$, Mike Thomas ${ }^{10}$, Steve Turner ${ }^{11}$, Nikolaos G. Papadopoulos ${ }^{1,12}$

1 Division of Infection, Immunity and Respiratory Medicine, School of Biological Sciences, Faculty of Biology, Medicine and Health, University of Manchester, NIHR Manchester Biomedical Research Centre, Manchester University Hospitals NHS Foundation Trust, Manchester Academic Health Science Centre, Manchester, UK.

2 Respiratory Effectiveness Group, Ely, UK.

${ }^{3}$ Respiratory Medicine, Sir Charles Gairdner Hospital, Perth, Australia and Medical School, Curtin University, Perth, Australia.

${ }^{4}$ Family Physician Airways Group of Canada, University of Toronto, ON, Canada.

${ }^{5}$ Respiratory Medicine, Dept of Medical Sciences, University of Ferrara, Ferrara, Italy.

${ }^{6}$ School of Medicine, University of Glasgow, Glasgow, UK.

7 Department of Pediatrics, Boston Children's Hospital, Boston, Mass.

8 Observational and Pragmatic Research Institute, Singapore, Singapore and Centre of Academic Primary Care, Division of Applied Health Sciences, University of Aberdeen, Aberdeen, UK

9 Department of Paediatrics, KK Women's and Children's Hospital, Singapore.

10 Primary Care and Population Sciences, Faculty of Medicine, University of Southampton, Southampton, UK.

${ }^{11}$ Child Health, University of Aberdeen, Aberdeen, UK.

${ }^{12}$ Allergy Department, 2nd Pediatric Clinic, National and Kapodistrian University of Athens, Athens, Greece.

\section{Correspondence}

Nikolaos G. Papadopoulos. Division of Infection, Immunity and Respiratory Medicine, University of Manchester, Royal Manchester Children's Hospital, Oxford Road, Manchester, M13 9WL, UK. Email nikolaos.papadopoulos@manchester.ac.uk

Word count: 3657 (excluding abstract and refs)

Tables/Figures: 4 figures/ 4 tables. ( 4 supplementary figures $\& 4$ supplementary tables)

Key words: asthma exacerbations; antibiotics; oral corticosteroids

Take Home Message: Antibiotics are regularly prescribed for asthma exacerbation, however, there is little clinical benefit of the routine addition of antibiotics to usual OCS treatment for managing asthma exacerbations in primary care patients. 
Abstract [250 words]

Background: Asthma exacerbations are major contributors to asthma morbidity and mortality. They are usually managed with bronchodilators and oral corticosteroids (OCS), but clinical trial evidence suggests antibiotics could be beneficial. We aimed to assess whether treatment of asthma exacerbations with antibiotics in addition to OCS improved outcomes in larger more representative routine care populations.

Method: A retrospective comparative effectiveness study into managing asthma exacerbations with OCS alone versus OCS plus antibiotics was conducted using the Optimum Patient Care Research Database. The dataset included 28,637 patients, following propensity score matching 20,024 adults and 4,184 children were analyzed.

Results: Antibiotics in addition to OCS were prescribed for the treatment of asthma exacerbations in $45 \%$ of adults and $32 \%$ of children.

Compared to OCS alone, OCS plus antibiotics was associated with reduced risk of having an asthma/wheeze consultation in the following 2 weeks (children HR 0.84 (95\% Cl 0.73-0.96), p=0.012; adults HR 0.86 (95\% $\mathrm{Cl} 0.81-0.91), p<0.001$ ), but an increase in risk of a further OCS prescription for a new/ongoing exacerbation within 6 weeks in adults (HR $1.11(95 \% \mathrm{Cl} 1.01-1.21), \mathrm{p}=0.030)$, but not children.

Penicillins, but not macrolides, were associated with a reduction in the odds of a subsequent asthma/wheeze consultation compared to OCS alone, in both adults and children.

Conclusion: Antibiotics were frequently prescribed in relation to asthma exacerbations, contrary to guideline recommendations. Overall, the routine addition of antibiotics to OCS in the management of asthma exacerbations appeared to confer little clinical benefit, especially when considering the risks of antibiotic overuse. 


\section{Introduction}

Asthma exacerbations are the major contributor to morbidity and mortality and a significant burden in terms of healthcare resource utilisation. Therefore, there is a need to optimise management approaches for asthma exacerbations. Respiratory viruses (especially rhinovirus) are the most common triggers of asthma exacerbations[1,2] but other factors can increase the risk/severity of exacerbations. Recent evidence suggests atypical bacterial infections may contribute to exacerbation severity.[3]

Standard management of asthma exacerbations involves the use of bronchodilators and, in the case of moderate to severe exacerbations, systemic steroids.[4,5] However, there is some evidence to suggest macrolide antibiotics and the ketolide antibiotic, telithromycin, may have a beneficial effect on asthma exacerbations through their antibacterial and/or anti-inflammatory properties.[3] A double-blind randomised controlled trial $(R C T)$ in adult patients $(n=278)$ with acute asthma exacerbations found a small but significant reduction in asthma symptoms among patients receiving add-on telithromycin compared with placebo.[6] $A$ second open-labelled randomised study found that in children with acute asthma $(n=40)$ the addition of clarithromycin may offer benefits over standard exacerbation treatment.[7] Current real-world evidence suggests that macrolide use has no significant benefit in acute asthma compared to other common antibiotics such as amoxicillin.[8] A recent Cochrane review found very limited evidence that antibiotics are beneficial to patients having asthma exacerbations, however, their conclusions were limited by a lack of studies.[9]

The RCT findings warrant further exploration in a larger more heterogeneous population that is representative of asthma patients who are routinely treated for their exacerbations in primary care. Therefore, we used realworld data to evaluate the comparative effectiveness of managing asthma exacerbations with a single acute course of oral corticosteroids (i.e. usual care) versus a single course of antibiotics in addition to oral corticosteroids, in adult and paediatric asthma populations.

\section{Methods}

\section{Study Design}

This is an observational primary care database study of the comparative effectiveness of treating patients experiencing an asthma exacerbation with a single course of antibiotics alongside oral corticosteroids (OCS) compared to the usual care of OCS alone.

\section{Data Sources and Permissions}

Historical electronic medical records from the Optimum Patient Care Research Database (OPCRD) were used. At the time of this study, the OPCRD contained anonymised, longitudinal medical records for approximately 6 million UK primary care patients, from more than 525 GP practices across the UK. The OPCRD is approved by the Trent Multi-Centre Research Ethics Committee for clinical research use. This study was approved by the Anonymised Data Ethics \& Protocol Transparency committee (ADEPT1519) and registered with the European Network of Centres for Pharmacoepidemiology and Pharmacovigilance (EUPAS 12132). We have followed the STROBE guidance for reporting observational evidence (strobestatement.org). 


\section{Patient population}

Patients were included if they had a prescription for OCS on the same date as a Read code for asthma or wheeze, which was taken to indicate an asthma exacerbation, between 1 January 2004 and 31 December 2014. Index Prescription Date (IPD) was the first date in this study period, when the patient received a prescription for OCS; patients were required to have had no OCS prescriptions (acute or maintenance doses) in the previous 6 months. Patients who received an acute course of OCS were compared to those who received a single acute course of antibiotics in addition to a prescription for OCS at IPD. The first OCS prescription was used so that the IPD represented the start of an exacerbation and not an ongoing exacerbation, and this reduced the chance of previous exacerbation treatment influencing treatment decisions at IPD. Patients were characterised over a 6 -month baseline period immediately prior to IPD and outcomes evaluated in the 12 weeks immediately post IPD (Figure 1).

Inclusion Criteria: aged 2-65 years at IPD; Read codes for asthma (or wheeze if $\leq 5$ years old) on $\geq 3$ occasions ever; $\geq 1$ Read code for asthma (or wheeze if $\leq 5$ years old) during baseline; $\geq 1$ inhaled corticosteroid (ICS) or LTRA prescription during baseline; $\geq 38$ weeks continuous records ( $\geq 26$ weeks prior to IPD and $\geq 12$ weeks following IPD).

Exclusion criteria: received regular antibiotics ( $>5$ prescriptions during baseline); had an additional chronic respiratory condition; aged $\geq 19$ years with a diagnosis of chronic obstructive pulmonary disease (COPD) (Supplementary figure 1).

\section{Outcomes}

The primary study endpoint was time to first primary care consultation coded for asthma/wheeze in the 2week outcome period.

Secondary outcomes were: time to first primary care consultation with a Read code for asthma/wheeze resulting in an OCS prescription with or without antibiotics in the 2-, 6- and 12-week periods post IPD and time to first hospitalisation and emergency department attendance for an exacerbation in the 2-, 6- and 12week periods post IPD.

Exploratory outcomes included the type of antibiotics prescribed at IPD (macrolides versus penicillins), blood eosinophil counts and outcomes in the different paediatric age groups (2-5, 6-12 and 13-18 years).

\section{Statistical analysis}

Data were separated into two age groups: paediatric patients (2-18 year olds) and adults (19-65 year olds). Demographics and clinical characteristics were compared between those given OCS and those given OCS plus antibiotics at IPD, using chi-squared tests. Backward stepwise multivariate logistic regression was used to determine the demographic and clinical characteristics that were predictors of a patient receiving OCS plus antibiotics.

To minimise confounding, individuals from the two groups (OCS plus antibiotics and OCS alone) were matched using 1-1 propensity score matching, using the nearest neighbour method and a caliper width of 0.25. The groups were matched on age, sex, Body Mass Index (BMI; or BMI z-scores in those under 18 year 
old as this gives a measure of relative weight adjusted for child age and sex), GINA category (based on 2018 guidelines[10]), season of IPD, smoking status, year of IPD and number of consultations for asthma/wheeze in the baseline period. Where matching variables (i.e. smoking status or BMI/zBMI) were missing an additional category for missing values was included; $29.1 \%(1,930 / 6,632)$ of children and $3.7 \%(818 / 22,005)$ of adults had at least one of these two variables missing. The time to primary care consultation for asthma/wheeze and time to primary care consultations for asthma/wheeze resulting in OCS were analysed using Cox proportional hazards regression. The number of patients with at least one primary care consultation and number of those with a respiratory related emergency department visit or hospitalisation were compared using chi-squared or Fisher's exact tests as appropriate. All analyses were performed with R software (www.r-project.org/). R packages used were Hmisc 4.2-0, Gmisc 1.8, htmlTable 1.13.1, survival 2.41-3, ggplot2 3.1.0, survminer 0.4.3.999, Matchlt 3.0.2, forcat 0.4.0, MASS v7.3-47 and the World Health Organisation macros igrowup_standard.r and who2007.r.

\section{Results}

28,637 patients fulfilled the eligibility criteria; 22,005 adults (19-65 years) and 6,632 children (2-18 years) (Supplementary figure 1). A large proportion of patients received antibiotics in addition to OCS for the treatment of asthma exacerbations at IPD; 10,012 (45\%) of adults and 2,094 (32\%) of children. There were significant differences in the demographic and clinical characteristics between those who received OCS plus antibiotics compared to those who received OCS alone (Supplementary tables 1-3). The odds of receiving an antibiotic were increased with age, being male, being a smoker or ex-smoker, presenting in winter or in more recent years, while the odds of receiving an antibiotic were decreased in children, those presenting in the summer, those with consultations resulting in a short-acting $\beta$-agonist (SABA) prescription in the previous 6 months or an active rhinitis diagnosis (Table 1).

Following matching, 20,024 (10,012 per group) adults and 4,184 (2,092 per group) children were included in subsequent analyses (Tables 2-3 and supplementary table 4 ).

\section{Consultations in the 2-, 6- and 12-week outcome period}

The addition of antibiotics to OCS is associated with a reduced risk of having an asthma/wheeze consultation in the following 2 weeks (children HR 0.84 (95\% Cl 0.73-0.96), p=0.012; adults HR 0.86 (95\% Cl 0.81-0.91), $\mathrm{p}<0.001$; Figures $2 a, b, 3)$. In the 2 weeks post-IPD $20.0 \%(2,001 / 10,012)$ of adults who received OCS plus antibiotics had a subsequent asthma/wheeze consultation compared to $22.9 \%(2,289 / 10,012)$ of those who received OCS alone $(p<0.001$, Supplementary figure 2$)$. Similarly, in children $19.6 \%(409 / 2,092)$ receiving OCS plus antibiotics compared to $22.8 \%(478 / 2,092)$ receiving OCS alone had a subsequent consultation within 2 weeks ( $p=0.010$, Supplementary figure 2 ). In the 2 weeks post IPD there was no difference in the time to first asthma/wheeze consultation resulting in a repeated OCS prescription with or without antibiotics, i.e. indicating a new or ongoing exacerbation, for either adults or children (children HR $0.92(95 \% \mathrm{Cl} 0.64-$ 1.33), $p=0.650$; adults HR 1.10 (95\% $\mathrm{Cl} 0.98-1.24), p=0.100)$. When prescription for OCS and/or antibiotics was used as the outcome at 2 weeks post IPD, there was no difference between the groups receiving OCS 
An exploratory analysis of effect of antibiotics in different paediatric age groups (2-5, 6-12 and 13-18 years) showed similar trends to the group as a whole (data available on request).

An exploratory analysis of adults with low blood eosinophil counts (0-0.2 109/L) compared to high blood eosinophil counts $\left(>0.210^{9} / \mathrm{L}\right)$ was conducted. The addition of antibiotics at IPD was significantly associated with a reduced risk of an asthma/wheeze consultation in the 2 weeks post IPD, which was of a similar magnitude in both those with high and with low blood eosinophil counts (High eos HR $0.87(95 \% \mathrm{Cl} 0.77$ 0.98), $p=0.018$; Low eos HR 0.84 (95\% Cl 0.75-0.94), p=0.003; Supplementary figure 4)). In both those with a high blood eosinophil count and a low blood eosinophil count there was no difference between the OCS and OCS plus antibiotic groups in the time to first asthma/wheeze consultation for OCS with or without antibiotics in the 2, 6 and 12 week outcome periods.was significantly increased in those who received antibiotics alongside OCS at IPD (2wk outcome HR 1.52 (95\% Cl 1.08-2.13), p=0.017; 6wk outcome HR 1.67 (95\% Cl 1.29-2.16), $p<0.001 ; 12$ wk outcome HR 1.68 (95\% Cl 1.36-2.06), $p<0.001)$. In those with low blood eosinophil counts the risk of a consultation for OCS with or without antibiotics in those who received antibiotics alongside OCS at IPD was only significantly increased in the 12 week outcome period and tended towards a lower magnitude increase (HR $1.33(95 \%$ Cl 1.07-1.65), $p=0.009$ ).

\section{Emergency department attendances and hospitalisations}

Only a small number of patients experienced a severe exacerbation, defined as requiring an emergency department attendance or hospitalisation $(<0.5 \%$ of patients had an emergency department attendance or hospitalisation in the 12 weeks post IPD) so Cox proportional hazards regression was not performed. There were no significant differences between the OCS plus antibiotics and OCS alone groups in the number of patients with an emergency department attendance or hospitalisation (Table 4). 


\section{Antibiotic type: Penicillins versus Macrolides}

In children given antibiotics at IPD, 86.1\% (1,802/2,092) received penicillins and 10.0\% $(210 / 2,092)$ received macrolides. Of those who received OCS plus penicillin, 19.0\% had an asthma/wheeze consultation in the 2 weeks post IPD, which was significantly less than in those who received OCS alone $(22.8 \%, p=0.004)$. However, in those given macrolides the percentage of children with an asthma/wheeze consultation in the first 2 weeks was not significantly different $(23.8 \%, p=0.82$, Figure $4 a)$ compared to OCS alone.

In the adults who received antibiotics at IPD $73.6 \%(7,371 / 10,012)$ received penicillins and $17.1 \%$ $(1,708 / 10,012)$ received macrolides. Similarly to in children, penicillins, but not macrolides, at IPD were associated with a significant reduction in the number of patients having a subsequent asthma/wheeze consultation in the 2 weeks post IPD compared to OCS alone (penicillins 19.1\% vs 22.9\% OCS alone, $p<0.001$; macrolides $21.8 \%$ vs $22.9 \%$ OCS alone, $p=0.37$, Figure $4 b$ ).

In both the paediatric and adult groups neither penicillins nor macrolides were associated with a significant difference in the number of patients having an asthma/wheeze consultation resulting in an OCS prescription with or without an antibiotic, in the 2- or 6-week outcome periods (children 2-week outcome p=0.33, 6-week outcome $p=0.68$; adults 2-week outcome $p=0.29$, 6-week outcome $p=0.16$; Figure 4a\&b).

\section{Discussion}

We have investigated the effectiveness of adding antibiotics alongside OCS for the treatment of asthma exacerbations in a heterogeneous real-life population comprising both adult and paediatric asthma patients. The addition of antibiotics to OCS is associated with a small reduction in the absolute risk of a subsequent asthma/wheeze consultation in the following 2 weeks; around 3\% fewer patients having consultations for asthma/wheeze. However, there was no difference in the rates of prescription of OCS and/or antibiotics at 2 weeks. One possible explanation for this is that GPs used a different read code at follow up at 2 weeks when further antibiotic treatment was prescribed. In contrast, in adults, but not children, there was a slightly increased risk of a consultation for a new/ongoing exacerbation (defined as a repeated OCS prescription) in the 6 weeks post IPD. The very low numbers of emergency department attendances and hospitalisations, which may be due partly to the poor recording of emergency department attendances and hospitalisations in primary care databases, make it difficult to draw firm conclusions. However, we saw no difference in the numbers of emergency department attendances or hospitalisations associated with the addition of antibiotics. While there were statistically significant differences, the magnitude was relatively small, and needs to be balanced against the adverse effects of antibiotic use, both at individual and at community level. The lack of impact on repeat prescription of OCS and/or antibiotics suggests that addition of antibiotics does not reduce treatment failure and thus healthcare resource utilisation. Our analysis occurred at group aggregated level, hence it is possible that while for most patients the addition of an antibiotic is of no benefit, there may be subgroups who benefit, and this should be a focus of further research. In a post hoc analysis looking at blood eosinophil levels we found no significant differences in the any of the outcomes between those with high blood eosinophil levels (>0.2x10\%/L) and those with low blood eosinophil counts. who received ocs and antibiotics at IPD actually had a significantly increased risk of subsequent consultations resulting in OCS treatment compared to those who received OCS alone, while in those with low eosinophil counts the risk was 
only increased in the 12 wk outcome period. In a primary care population, the routine addition of antibiotics appears to be of minimal, if any, clinical benefit in treating asthma exacerbations, especially when considering the major risk of antibiotic resistance associated with antibiotic overuse [11].

The small increase in time until a subsequent asthma/wheeze consultation in patients prescribed antibiotics may be partly explained by patients receiving antibiotics feeling that their expectations have been met, making them less likely to return for further treatment for ongoing symptoms. A course of antibiotics will likely last for 5-7 days, compared to the usual shorter course of OCS, so it could be expected that patients prescribed antibiotics who have ongoing symptoms are going to finish the longer course of antibiotics, before returning for a subsequent consultation. A limitation of this study is that we do not have information regarding delayed prescribing, as this is not well recorded in primary care databases. A previous study in UK primary care has suggested around $18 \%$ of antibiotic prescribing for lower respiratory tract infections (LRTI) in adults may be delayed prescribing, where patients are advised to take one treatment first followed by the second if symptoms are unresolved [12]. Therefore, in patients who received both OCS and antibiotics at IPD the time until those who have ongoing symptoms return for a subsequent consultation could be extended, biasing the primary outcome to favour OCS and antibiotics at IPD. While antibiotics may reduce the chances of patients returning with a LRTI, those with LRTIs are at increased risk of having an exacerbation [13]. This may in part explain why we observed an increased risk of exacerbations at 6 weeks in the antibiotic treated adult population. Although we matched our patient groups for a number of variables there is the potential for residual confounding. The higher number of co-morbidities in the adult population receiving OCS plus antibiotics may have influenced the prescribing at 2 and 6 weeks if symptoms had not fully resolved. There may have been other factors, such as positive sputum cultures, that guided treatment decisions which are not well recorded within the database. Time to the first primary care consultation for asthma/wheeze was only analysed at 2 weeks post IPD; this outcome included all consultations with an asthma or wheeze Read code. It was felt patients returning within 2 weeks most likely represent those with ongoing exacerbations rather than routine/follow-up appointments, however, it is possible for both groups that some planned routine appointments are included within this outcome. A further limitation is that we required an asthma/wheeze Read code at follow up, however, analysis of a very small random subset $(0.1 \%$ of the sample size) suggests we have missed at least $7.5 \%$ of respiratory related consultations at 2 weeks post IPD, as other Read codes (e.g. for chest infection) were used.

Despite some RCTs suggesting a beneficial effect of macrolide antibiotics in both treating and preventing exacerbations,[6,7,14] there are a number of studies that have found no benefit in the use of antibiotics in adults receiving hospital treatment for asthma exacerbations. A retrospective cohort study of adult asthmatics hospitalised for asthma exacerbations found an increase in the length of hospital stay in those prescribed antibiotics.[15] A RCT of adult asthmatics hospitalised with asthma exacerbations found amoxicillin compared to placebo had no significant effect on length of hospital stay, symptoms or lung function.[16] Similarly, azithromycin compared to placebo had no significant effect on quality-of-life questionnaire scores, lung function and symptom score in adult asthmatics presenting with asthma exacerbations in secondary care.[17] 
Our study benefits from a large heterogeneous real-life population that includes both paediatric and adult patients and addresses an important need in assessing antibiotic use in asthma exacerbations, as highlighted by a recent Cochrane review.[9] The mixed population of patients included represent the asthmatic population typically seen in primary care, where most asthma exacerbations are treated, and where it can be difficult to separate what is a non-infective asthma exacerbation and what is a (mostly viral) infection. It can be difficult to distinguish between a non-infective asthma exacerbation and LRTI as the symptoms are often indistinguishable, particularly, but not exclusively, in those with a previous history of asthma.[18] Furthermore, exacerbations and infections are not independent events; respiratory infections are a major trigger of asthma exacerbations.[19] However, viral infections are thought to trigger up to $85 \%$ of acute asthma exacerbations in children and about 60\% in adults.[20] Bacterial infections are only thought to be responsible for a minority of exacerbations, thus little or no effect of antibiotics would likely be expected. It is possible some of the patients included may have had COPD rather than, or alongside, asthma, particularly in the OCS plus antibiotic group where the number of current smokers is higher. However, in a sub-analysis of patients under and over 40 years of age, where the risk of COPD is increased, no differences were found between the two groups.

We found high levels of antibiotic prescribing, which is perhaps surprising given the addition of antibiotics is currently not recommended within the guidelines for the treatment of asthma exacerbations.[4] Antibiotics may be prescribed due to the uncertainties around the definition and symptoms of asthma exacerbations and there being multiple potential causes of the increased respiratory symptoms, for some of which antibiotics may be beneficial. It is possible some of the antibiotic prescribing at IPD could be for co-morbidities; as this is a real-life population some patients may have presented with other infections, for example otitis media, that prompted the antibiotic prescription, alongside symptoms of an asthma exacerbation. Information on such comorbidities was not collected, but many of the other potential diagnoses/infections would likely be of viral origin. The level of antibiotic prescribing observed here was similar to that reported in previous studies. A $1992 / 1993$ study found that approximately $40 \%$ of asthmatic patients experiencing an exacerbation managed in UK primary care, were given antibiotics.[21] In another study $44.6 \%$ of adult asthmatics seeking emergency treatment for an asthma exacerbation had received antibiotics in the previous 4 weeks.[17] Antibiotic prescribing was more common in certain groups: older people, males, smokers or ex-smokers, and was more common in winter, and interestingly increased between 2004 and 2014. The increase in antibiotic prescribing could be due to increased time pressures, reduced access to GP appointments over this period, related to increased concern about the consequences of missing something or not meeting increased patient/carer expectations [22,23,24].

Patients prescribed penicillins alongside OCS had a small reduction in the odds of a subsequent asthma/wheeze consultation compared to OCS alone. This is consistent with a previous study of penicillin use in asthma[8] and studies that have found penicillin treatment for COPD exacerbations, and for LRTIs in patients without respiratory disease, is associated with a lower risk of needing repeat antibiotics.[20,21] In those prescribed macrolides alongside OCS the odds of a subsequent asthma/wheeze consultation were not significantly different compared to those receiving OCS alone. Hence the observed statistically significant benefit was associated with only penicillins, not macrolides. This apparent benefit with penicillins could be an artefact of the GPs choosing to prescribe macrolides to those with more severe illness that they may have 
felt would not be adequately treated with penicillins. This could explain the divergence This contrasts-with previous RCTs that found beneficial effects of macrolides[6,7], although it should be noted it is difficult to draw firm conclusions from our study given the number of patients prescribed macrolides is relatively low. The patients in our study and in other studies where the beneficial effect of penicillins have been seen[8,25,26] have presented in primary care, whereas the studies showing macrolide benefits have been in patients that have presented in the emergency department.[6,7]. Patients attending the emergency department may have different underlying disease severity or a different microbiome that makes macrolides more effective in that scenario.

In conclusion, we found antibiotic use to be common in asthma exacerbations but did not find clear evidence of a clinically significant benefit of the addition of antibiotics to usual care.

\section{Acknowledgements}

This project was supported by the Respiratory Effectiveness Group. Data and data management support was provided in-kind by Optimum Patient Care (www.opcrd.co.uk) and Derek Skinner at OPC. Clare Murray is supported by the NIHR Manchester Biomedical Research Centre.

\section{References}

1. Korppi M. Management of bacterial infections in children with asthma. Expert Rev Anti Infect Ther. 2009; 7:869-77.

2. Nicholson KG, Kent J, Ireland DC. Respiratory viruses and exacerbations of asthma in adults. BMJ. 1993; 307: 982-986.

3. Johnston SL. Macrolide antibiotics and asthma treatment. J Allergy Clin Immunol. 2006; 117:1233-6.

4. Global Initiative for Asthma (GINA). Pocket Guide for Asthma Management and Prevention. Updated 2019. Available online at: https://ginasthma.org/wp-content/uploads/2019/04/GINA-2019-main-PocketGuide-wms.pdf (last accessed: 16/09/19)

5. Reddel, HK, Taylor DR, Bateman ED, Boulet L-P, Boushey HA, Busse WW, Casale TB, Chanez P, Enright PL, Gibson PG, de Jongste JC, Kerstjens HAM, Lazarus SC, Levy ML, O’Byrne PM, Partridge MR, Pavord ID, Sears MR, Sterk PJ, Stoloff SW, Sullivan SD, Szefler SJ, Thomas MD, \& Wenzel SE, on behalf of the American Thoracic Society/European Respiratory Society Task Force on Asthma Control and Exacerbations. An official American Thoracic Society/European Respiratory Society statement: asthma control and exacerbations: standardizing endpoints for clinical asthma trials and clinical practice. Am J Respir Crit Care Med. 2009; 180:59-99, 2009.

6. Johnston SL, Blasi F, Black PN, Martin RJ, Farrell DJ, Nieman RB; TELICAST Investigators. The effect of telithromycin in acute exacerbations of asthma. N Engl J Med. 2006; 354:1589-600.

7. Koutsoubari I, Papaevangelou V, Konstantinou GN, Makrinioti H, Xepapadaki P, Kafetzis D, Papadopoulos NG. Effect of clarithromycin on acute asthma exacerbations in children: an open randomized study. Pediatr Allergy Immunol. 2012; 23:385-90.

8. Stolbrink M, Bonnett LJ, Blakey JD. Antibiotic Choice and Duration Associate with Repeat Prescriptions in Infective Asthma Exacerbations. J Allergy Clin Immunol Pract. 2019; 7:548 - 553.

9. Normansell, R., Sayer, B., Waterson, S., Dennett, E.J., Del Forno, M., and Dunleavy, A. Antibiotics for exacerbations of asthma. Cochrane Database Syst Rev. 2018; 6: CD002741.

10. Global Initiative for Asthma (GINA). Pocket Guide for Asthma Management and Prevention. Updated 2018. Available online at: https://ginasthma.org/wp-content/uploads/2018/03/wms-GINA-main-pocketguide 2018-v1.0.pdf (last accessed: 8/3/2020)

11. World Health Organization. Antimicrobial resistance - fact sheet. 2018 https://www.who.int/newsroom/fact-sheets/detail/antimicrobial-resistance (last accessed: 19/5/2020) 
12. Little P, Stuart B, Smith S, Thompson MJ, Knox K, van den Bruel A et al. Antibiotic prescription strategies and adverse outcome for uncomplicated lower respiratory tract infections: prospective cough complication cohort (3C) study BMJ 2017; 357 :j2148

13. Price D, Wilson AM, Chisholm, A, Rigazio A, Burden A, Thomas M, King C. Predicting frequent asthma exacerbations using blood eosinophil count and other patient data routinely available in clinical practice. Journal of Asthma and Allergy. 2016; 9:1-12.

14. Gibson PG, Yang IA, Upham JW, Reynolds PN, Hodge S, James AL, Jenkins C, Peters MJ, Marks GB, Baraket M, Powell H, Taylor SL, Leong LEX, Rogers GB \& Simpson JL. Effect of azithromycin on asthma exacerbations and quality of life in adults with persistent uncontrolled asthma (AMAZES): a randomised, double-blind, placebo-controlled trial. The Lancet. 2017; 390:659-668.

15. Stefan MS, Shieh MS, Spitzer KA, Pekow PS, Krishnan, JA, \& Au DH, Lindenauer PK. Association of Antibiotic Treatment With Outcomes in Patients Hospitalized for an Asthma Exacerbation Treated With Systemic Corticosteroids. JAMA Internal Medicine. 2019;179(3):333-339.

16. Graham VAL, Knowles GK, Milton AF, Davies, RJ. Routine Antibiotics in Hospital Management of Acute Asthma. The Lancet. 1982; 319:418-421.

17. Johnston, SL, Szigeti M, Cross M, Brightling C, Chaudhuri R, Harrison T, Mansur A, Robison L, Sattar Z, Jackson D, Mallia P, Wong E, Corrigan C, Higgins B, Ind P Singh D, Thomson NC, Ashby D, Chauhan A; For the AZALEA Trial Team Azithromycin for Acute Exacerbations of Asthma: The AZALEA Randomized Clinical Trial. JAMA Intern Med. 2016; 176:1630-1637.

18. Guibas GV, Tsolia M, Christodoulou I, Stripeli F, Sakkou Z, Papadopoulos NG. Distinction between rhinovirus-induced acute asthma and asthma-augmented influenza infection. Clin Exp Allergy. 2018; 48(5):536-543.

19. Xepapdaki P, Megremis S, Kitsioulis NA, Papadopoulos NG. Infections in the nose and exacerbations of chronic respiratory disorders. In: Bachert C, Bourdin A, Chanez P, eds. The Nose and Sinuses in Respiratory Disorders (ERS Monograph). Shefield, European Respiratory Society, 2017; pp000-000.

20. Saraya T, Kurai D, Ishii H, Ito A, Sasaki Y, Niwa S, Kiyota N, Tsukagoshi H, Kozawa K, Goto $\mathrm{H}$, Takizawa $\mathrm{H}$. Epidemiology of virus-induced asthma exacerbations: with special reference to the role of human rhinovirus. Front Microbiol. 2014; 5: 226.

21. Neville RG, Hoskins G, Smith B, Clark RA. How general practitioners manage acute asthma attacks. Thorax. 1997; 52:153-156.

22. Ashworth $M$, White $P$, Jongsma $H$, Schofield $P$, Armstrong $D$. Antibiotic prescribing and patient satisfaction in primary care in England: cross-sectional analysis of national patient survey data and prescribing data. Br J Gen Pract. 2016;66(642):e40-e46. doi:10.3399/bjgp15X688105

23. SernaMC, Real J, Ribes E et al. Factors determining antibiotic prescription in primary care. Enferm Infecc Microbiol Clin 2011; 29: 193-200.

24. Lucas PJ, Cabral C, Hay AD, Horwood J. A systematic review of parent and clinician views and perceptions that influence prescribing decisions in relation to acute childhood infections in primary care. Scand J Prim Health Care. 2015;33(1):11-20. doi:10.3109/02813432.2015.1001942

25. Stolbrink M, Bonnett LJ, Blakey JD. Antibiotics for COPD exacerbations: does drug or duration matter? A primary care database analysis. BMJ Open Resp Res. 2019; 6:e00458.

26. Stolbrink M, Bonnett LJ, Blakey JD. Amoxicillin is associated with a lower risk of further antibiotic prescriptions for lower respiratory tract infections in primary care - A database analysis spanning over 30 years. Eur Clin Respir J. 2018; 5(1): 1529535. 


\section{Tables}

Table 1. Predictors of receiving Oral Corticosteroids plus antibiotics at Index Prescription Date (IPD).

\begin{tabular}{lrrr}
\hline & Odds ratio & $\mathbf{9 5 \%}$ Cl & p-value \\
\hline Age & & & \\
$2-5$ yrs & 0.80 & $(0.67-0.95)$ & 0.0126 \\
$6-12$ yrs & 0.75 & $(0.66-0.85)$ & $<0.0001$ \\
$13-18$ yrs & 0.91 & $(0.80-1.04)$ & 0.1526 \\
$19-25$ yrs & REF & & \\
$26-35$ yrs & 1.07 & $(0.96-1.20)$ & 0.2305 \\
$36-45$ yrs & 1.18 & $(1.06-1.31)$ & 0.0026 \\
$46-55$ yrs & 1.38 & $(1.24-1.54)$ & $<0.0001$ \\
$56-65$ yrs & 1.62 & $(1.45-1.80)$ & $<0.0001$ \\
Male & 1.10 & $(1.04-1.15)$ & $<0.0003$ \\
Current Smoker & 1.56 & $(1.46-1.67)$ & $<0.0001$ \\
Ex-Smoker & 1.09 & $(1.03-1.17)$ & 0.0051 \\
Obese & 1.06 & $(1.00-1.13)$ & 0.0500 \\
Summer IPD & 0.82 & $(0.76-0.88)$ & $<0.0001$ \\
Autumn IPD & 1.08 & $(1.01-1.16)$ & 0.0210 \\
Winter IPD & 1.26 & $(1.18-1.35)$ & $<0.0001$ \\
IPD 2004-2007 & REF & & \\
IPD 2007-2009 & 1.18 & $(1.11-1.25)$ & $<0.0001$ \\
IPD 2010-2012 & 1.42 & $(1.33-1.51)$ & $<0.0001$ \\
IPD 2013-2014 & 1.55 & $(1.43-1.69)$ & $<0.0001$ \\
1 SABA consult in baseline & 0.95 & $(0.90-1.00)$ & 0.0373 \\
2 SABA consults in baseline & 0.88 & $(0.81-0.95)$ & 0.0019 \\
Active rhinitis & 0.90 & $(0.84-0.96)$ & 0.0025 \\
\hline SABA: Short-Acting Beta Agonist & & &
\end{tabular}

SABA: Short-Acting Beta Agonist 
Table 2. Demographic and clinical characteristics for 2-18 year olds, following propensity score matching. Values are $n(\%)$.

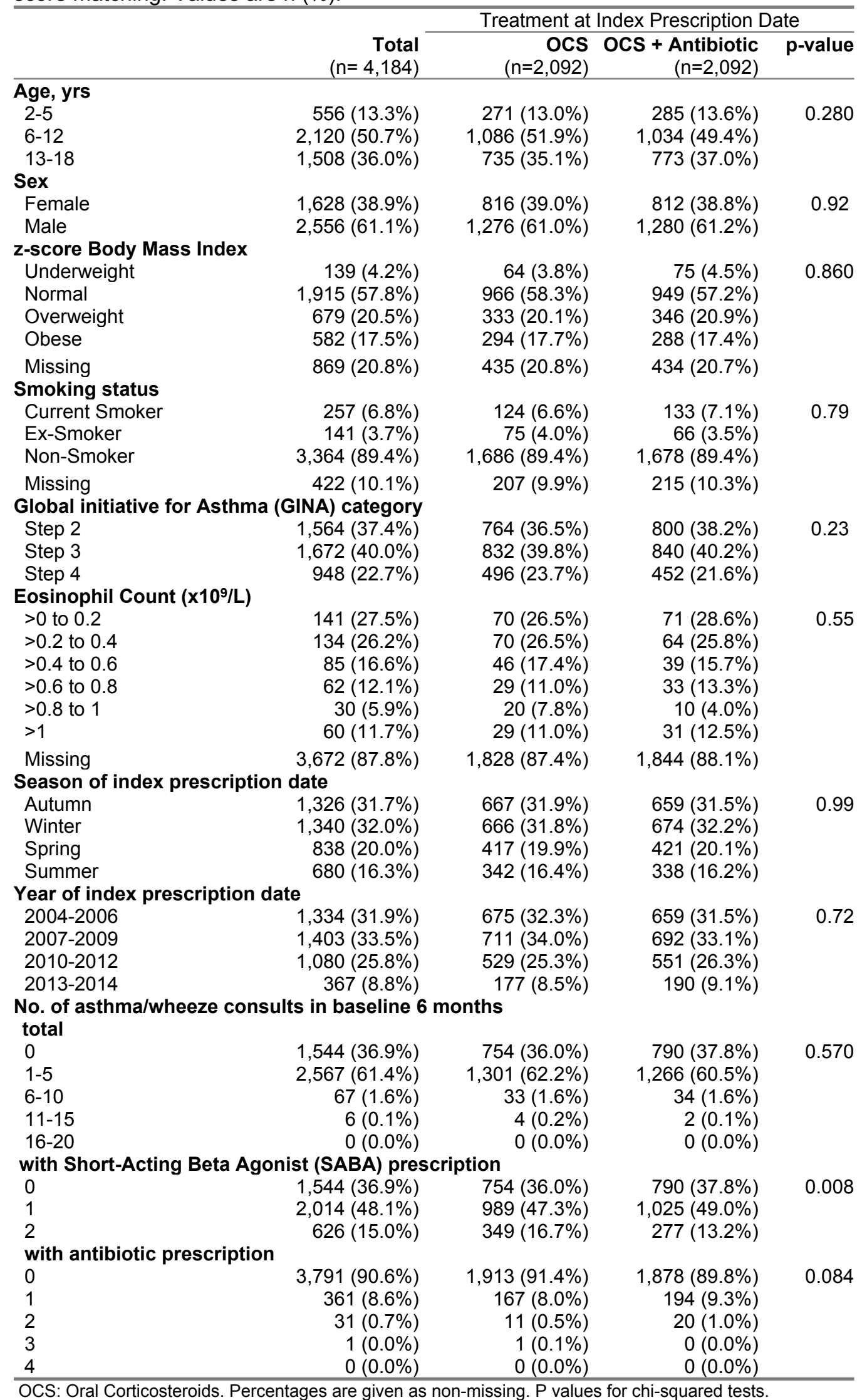


Table 3. Demographic and clinical characteristics for 19-65 year olds, following propensity score matching. Values are $\mathrm{n}(\%)$.

\begin{tabular}{|c|c|c|c|c|}
\hline & & \multicolumn{3}{|c|}{ Treatment at Index Prescription Date } \\
\hline & $\begin{array}{r}\text { Total } \\
(n=20,024)\end{array}$ & $\begin{array}{r}\text { OCS } \\
(n=10,012)\end{array}$ & $\begin{array}{r}\text { OCS + Antibiotic } \\
(\mathrm{n}=10,012)\end{array}$ & p-value \\
\hline \multicolumn{5}{|l|}{ Age, yrs } \\
\hline $19-25$ & $1,619(8.1 \%)$ & $839(8.4 \%)$ & $780(7.8 \%)$ & 0.003 \\
\hline $26-35$ & $3,334(16.7 \%)$ & $1,718(17.2 \%)$ & $1,616(16.1 \%)$ & \\
\hline $36-45$ & $5,099(25.5 \%)$ & $2,600(26.0 \%)$ & $2,499(25.0 \%)$ & \\
\hline $46-55$ & $5,110(25.5 \%)$ & $2,523(25.2 \%)$ & $2,587(25.8 \%)$ & \\
\hline $56-65$ & $4,862(24.3 \%)$ & $2,332(23.3 \%)$ & $2,530(25.3 \%)$ & \\
\hline \multicolumn{5}{|l|}{ Sex } \\
\hline Female & $12,970(64.8 \%)$ & $6,521(65.1 \%)$ & $6,449(64.4 \%)$ & 0.290 \\
\hline Male & $7,054(35.2 \%)$ & $3,491(34.9 \%)$ & $3,563(35.6 \%)$ & \\
\hline \multicolumn{5}{|l|}{ Body Mass Index } \\
\hline Underweight & 330 (1.7\%) & 165 (1.7\%) & 165 (1.7\%) & 0.900 \\
\hline Normal & $5,114(26.1 \%)$ & $2,578(26.3 \%)$ & $2,536(25.9 \%)$ & \\
\hline Overweight & $6,327(32.3 \%)$ & $3,174(32.4 \%)$ & $3,153(32.2 \%)$ & \\
\hline Obese & $7,835(40.0 \%)$ & $3,892(39.7 \%)$ & $3,943(40.2 \%)$ & \\
\hline Missing & $418(2.1 \%)$ & $203(2.0 \%)$ & $215(2.1 \%)$ & \\
\hline \multicolumn{5}{|l|}{ Smoking status } \\
\hline Current Smoker & $4,738(24.1 \%)$ & $2,219(22.5 \%)$ & $2,519(25.6 \%)$ & $<0.001$ \\
\hline Ex-Smoker & $5,323(27.0 \%)$ & $2,673(27.2 \%)$ & $2,650(26.9 \%)$ & \\
\hline Non-Smoker & $9,637(48.9 \%)$ & $4,950(50.3 \%)$ & $4,687(47.6 \%)$ & \\
\hline Missing & $326(1.6 \%)$ & $170(1.7 \%)$ & $156(1.6 \%)$ & \\
\hline \multicolumn{5}{|c|}{ Global initiative for Asthma (GINA) category } \\
\hline Step 2 & $5,903(29.5 \%)$ & $2,949(29.5 \%)$ & $2,954(29.5 \%)$ & 1.000 \\
\hline Step 3 & $5,552(27.7 \%)$ & $2,777(27.7 \%)$ & $2,775(27.7 \%)$ & \\
\hline Step 4 & $8,569(42.8 \%)$ & $4,286(42.8 \%)$ & $4,283(42.8 \%)$ & \\
\hline \multicolumn{5}{|c|}{ Eosinophil Count (x109/L) } \\
\hline$>0$ to 0.2 & $5,199(48.2 \%)$ & $2,607(48.5 \%)$ & $2,592(47.9 \%)$ & 0.26 \\
\hline$>0.2$ to 0.4 & $3,645(33.8 \%)$ & $1,804(33.6 \%)$ & $1,841(34.0 \%)$ & \\
\hline$>0.4$ to 0.6 & $1,275(11.8 \%)$ & $610(11.4 \%)$ & $665(12.3 \%)$ & \\
\hline$>0.6$ to 0.8 & $397(3.7 \%)$ & $217(4.0 \%)$ & $180(3.3 \%)$ & \\
\hline$>0.8$ to 1 & $152(1.4 \%)$ & $79(1.5 \%)$ & $73(1.3 \%)$ & \\
\hline$>1$ & $115(1.1 \%)$ & $55(1.0 \%)$ & $60(1.1 \%)$ & \\
\hline Missing & $9,241(46.1 \%)$ & $4,640(46.3 \%)$ & $4,601(46.0 \%)$ & \\
\hline \multicolumn{5}{|c|}{ Season of Index Prescription Date } \\
\hline Autumn & $5,334(26.6 \%)$ & $2,689(26.9 \%)$ & $2,645(26.4 \%)$ & 0.002 \\
\hline Winter & $6,772(33.8 \%)$ & $3,265(32.6 \%)$ & $3,507(35.0 \%)$ & \\
\hline Spring & $4,349(21.7 \%)$ & $2,204(22.0 \%)$ & $2,145(21.4 \%)$ & \\
\hline Summer & $3,569(17.8 \%)$ & 1,854 (18.5\%) & 1,715 (17.1\%) & \\
\hline \multicolumn{5}{|c|}{ Year of Index Prescription Date } \\
\hline $2004-2006$ & $5,668(28.3 \%)$ & $2,938(29.3 \%)$ & $2,730(27.3 \%)$ & $<0.001$ \\
\hline $2007-2009$ & $6,524(32.6 \%)$ & $3,325(33.2 \%)$ & $3,199(32.0 \%)$ & \\
\hline $2010-2012$ & $5,395(26.9 \%)$ & $2,621(26.2 \%)$ & $2,774(27.7 \%)$ & \\
\hline $2013-2014$ & $2,437(12.2 \%)$ & $1,128(11.3 \%)$ & $1,309(13.1 \%)$ & \\
\hline \multicolumn{5}{|c|}{$\begin{array}{l}\text { No. of asthma/wheeze consults in baseline } 6 \text { months } \\
\text { total }\end{array}$} \\
\hline 0 & $9,537(47.6 \%)$ & $4,716(47.1 \%)$ & $4,821(48.2 \%)$ & 0.420 \\
\hline $1-5$ & $10,176(50.8 \%)$ & $5,149(51.4 \%)$ & $5,027(50.2 \%)$ & \\
\hline $6-10$ & $272(1.4 \%)$ & $128(1.3 \%)$ & $144(1.4 \%)$ & \\
\hline $11-15$ & $37(0.2 \%)$ & $18(0.2 \%)$ & $19(0.2 \%)$ & \\
\hline $16-20$ & $2(0.0 \%)$ & $1(0.0 \%)$ & $1(0.0 \%)$ & \\
\hline $26-30$ & $0(0.0 \%)$ & $0(0.0 \%)$ & $0(0.0 \%)$ & \\
\hline \multicolumn{5}{|c|}{ with Short-Acting Beta Agonist (SABA) prescription } \\
\hline 0 & $9,537(47.6 \%)$ & $4,716(47.1 \%)$ & $4,821(48.2 \%)$ & 0.220 \\
\hline 1 & $8,697(43.4 \%)$ & $4,375(43.7 \%)$ & $4,322(43.2 \%)$ & \\
\hline 2 & $1,790(8.9 \%)$ & $921(9.2 \%)$ & $869(8.7 \%)$ & \\
\hline \multicolumn{5}{|c|}{ with antibiotic prescription } \\
\hline 0 & $18,330(91.5 \%)$ & $9,125(91.1 \%)$ & $9,205(91.9 \%)$ & 0.220 \\
\hline 1 & $1,534(7.7 \%)$ & $804(8.0 \%)$ & $730(7.3 \%)$ & \\
\hline 2 & $134(0.7 \%)$ & $68(0.7 \%)$ & $66(0.7 \%)$ & \\
\hline 3 & $21(0.1 \%)$ & $11(0.1 \%)$ & $10(0.1 \%)$ & \\
\hline 4 & $5(0.0 \%)$ & $4(0.0 \%)$ & $1(0.0 \%)$ & \\
\hline
\end{tabular}


Table 4. Number of patients with at least one severe exacerbation

\begin{tabular}{|c|c|c|c|c|c|c|c|}
\hline \multirow{2}{*}{$\begin{array}{l}\text { Outcome } \\
\text { period }\end{array}$} & & \multicolumn{3}{|c|}{$2-18$ year olds } & \multicolumn{3}{|c|}{$19-65$ year olds } \\
\hline & & $\begin{array}{r}\text { OCS } \\
(n=2,092)\end{array}$ & $\begin{array}{r}\text { OCS + } \\
\text { Antibiotic } \\
(n=2,092)\end{array}$ & $p$-value & $\begin{array}{r}\text { OCS } \\
(n=10,012)\end{array}$ & $\begin{array}{r}\text { OCS + } \\
\text { Antibiotic } \\
(n=10,012)\end{array}$ & -value \\
\hline \multirow[t]{2}{*}{2 weeks } & Emergency department visit & $4(0.2 \%)$ & $2(0.1 \%)$ & 0.69 & $20(0.2 \%)$ & $22(0.2 \%)$ & 0.88 \\
\hline & Hospitalisation & $3(0.1 \%)$ & $5(0.2 \%)$ & 0.73 & $22(0.2 \%)$ & $24(0.2 \%)$ & 0.88 \\
\hline \multirow[t]{2}{*}{6 weeks } & Emergency department visit & $7(0.3 \%)$ & $5(0.2 \%)$ & 0.77 & $33(0.3 \%)$ & $37(0.4 \%)$ & 0.72 \\
\hline & Hospitalisation & $9(0.4 \%)$ & $6(0.3 \%)$ & 0.61 & $35(0.3 \%)$ & $31(0.3 \%)$ & 0.71 \\
\hline \multirow[t]{2}{*}{12 weeks } & Emergency department visit & $11(0.5 \%)$ & $9(0.4 \%)$ & 0.82 & $51(0.5 \%)$ & $54(0.5 \%)$ & 0.84 \\
\hline & Hospitalisation & $12(0.6 \%)$ & $7(0.3 \%)$ & 0.36 & $44(0.4 \%)$ & $48(0.5 \%)$ & 0.75 \\
\hline
\end{tabular}




\section{A real-life comparative effectiveness study into the addition of antibiotics to the management of asthma exacerbations in primary care}

Clare S Murray ${ }^{1}$, Sarah J Lucas², John Blakey ${ }^{3}$, Alan Kaplan ${ }^{4}$, Alberto Papi ${ }^{5}$, James Paton ${ }^{6}$, Wanda

Phipatanakul $^{7}$, David Price ${ }^{8}$, Oon Hoe Teoh ${ }^{9}$, Mike Thomas ${ }^{10}$, Steve Turner ${ }^{11}$, Nikolaos G. Papadopoulos ${ }^{1,} 12$

1 Division of Infection, Immunity and Respiratory Medicine, School of Biological Sciences, Faculty of Biology, Medicine and Health, University of Manchester, NIHR Manchester Biomedical Research Centre, Manchester University Hospitals NHS Foundation Trust, Manchester Academic Health Science Centre, Manchester, UK.

2 Respiratory Effectiveness Group, Ely, UK.

${ }^{3}$ Respiratory Medicine, Sir Charles Gairdner Hospital, Perth, Australia and Medical School, Curtin University, Perth, Australia.

${ }^{4}$ Family Physician Airways Group of Canada, University of Toronto, ON, Canada.

${ }^{5}$ Respiratory Medicine, Dept of Medical Sciences, University of Ferrara, Ferrara, Italy.

${ }^{6}$ School of Medicine, University of Glasgow, Glasgow, UK.

7 Department of Pediatrics, Boston Children's Hospital, Boston, Mass.

8 Observational and Pragmatic Research Institute, Singapore, Singapore and Centre of Academic Primary Care, Division of Applied Health Sciences, University of Aberdeen, Aberdeen, UK

9 Department of Paediatrics, KK Women's and Children's Hospital, Singapore.

10 Primary Care and Population Sciences, Faculty of Medicine, University of Southampton, Southampton, UK.

${ }^{11}$ Child Health, University of Aberdeen, Aberdeen, UK.

${ }^{12}$ Allergy Department, 2nd Pediatric Clinic, National and Kapodistrian University of Athens, Athens, Greece.

\section{Correspondence}

Nikolaos G. Papadopoulos. Division of Infection, Immunity and Respiratory Medicine, University of Manchester, Royal Manchester Children's Hospital, Oxford Road, Manchester, M13 9WL, UK. Email nikolaos.papadopoulos@manchester.ac.uk

Word count: 3744 (excluding abstract and refs)

Tables/Figures: 4 figures/ 4 tables. ( 4 supplementary figures $\& 4$ supplementary tables)

Key words: asthma exacerbations; antibiotics; oral corticosteroids

Take Home Message: Antibiotics are regularly prescribed for asthma exacerbation, however, there is little clinical benefit of the routine addition of antibiotics to usual OCS treatment for managing asthma exacerbations in primary care patients. 
Abstract [250 words]

Background: Asthma exacerbations are major contributors to asthma morbidity and mortality. They are usually managed with bronchodilators and oral corticosteroids (OCS), but clinical trial evidence suggests antibiotics could be beneficial. We aimed to assess whether treatment of asthma exacerbations with antibiotics in addition to OCS improved outcomes in larger more representative routine care populations.

Method: A retrospective comparative effectiveness study into managing asthma exacerbations with OCS alone versus OCS plus antibiotics was conducted using the Optimum Patient Care Research Database. The dataset included 28,637 patients, following propensity score matching 20,024 adults and 4,184 children were analyzed.

Results: Antibiotics in addition to OCS were prescribed for the treatment of asthma exacerbations in $45 \%$ of adults and $32 \%$ of children.

Compared to OCS alone, OCS plus antibiotics was associated with reduced risk of having an asthma/wheeze consultation in the following 2 weeks (children HR 0.84 (95\% Cl 0.73-0.96), p=0.012; adults HR 0.86 (95\% $\mathrm{Cl} 0.81-0.91), p<0.001)$, but an increase in risk of a further OCS prescription for a new/ongoing exacerbation within 6 weeks in adults (HR $1.11(95 \% \mathrm{Cl} 1.01-1.21), p=0.030)$, but not children.

Penicillins, but not macrolides, were associated with a reduction in the odds of a subsequent asthma/wheeze consultation compared to OCS alone, in both adults and children.

Conclusion: Antibiotics were frequently prescribed in relation to asthma exacerbations, contrary to guideline recommendations. Overall, the routine addition of antibiotics to OCS in the management of asthma exacerbations appeared to confer little clinical benefit, especially when considering the risks of antibiotic overuse. 


\section{Introduction}

Asthma exacerbations are the major contributor to morbidity and mortality and a significant burden in terms of healthcare resource utilisation. Therefore, there is a need to optimise management approaches for asthma exacerbations. Respiratory viruses (especially rhinovirus) are the most common triggers of asthma exacerbations[1,2] but other factors can increase the risk/severity of exacerbations. Recent evidence suggests atypical bacterial infections may contribute to exacerbation severity.[3]

Standard management of asthma exacerbations involves the use of bronchodilators and, in the case of moderate to severe exacerbations, systemic steroids.[4,5] However, there is some evidence to suggest macrolide antibiotics and the ketolide antibiotic, telithromycin, may have a beneficial effect on asthma exacerbations through their antibacterial and/or anti-inflammatory properties.[3] A double-blind randomised controlled trial $(R C T)$ in adult patients $(n=278)$ with acute asthma exacerbations found a small but significant reduction in asthma symptoms among patients receiving add-on telithromycin compared with placebo.[6] A second open-labelled randomised study found that in children with acute asthma $(n=40)$ the addition of clarithromycin may offer benefits over standard exacerbation treatment.[7] Current real-world evidence suggests that macrolide use has no significant benefit in acute asthma compared to other common antibiotics such as amoxicillin.[8] A recent Cochrane review found very limited evidence that antibiotics are beneficial to patients having asthma exacerbations, however, their conclusions were limited by a lack of studies.[9]

The RCT findings warrant further exploration in a larger more heterogeneous population that is representative of asthma patients who are routinely treated for their exacerbations in primary care. Therefore, we used realworld data to evaluate the comparative effectiveness of managing asthma exacerbations with a single acute course of oral corticosteroids (i.e. usual care) versus a single course of antibiotics in addition to oral corticosteroids, in adult and paediatric asthma populations.

\section{Methods}

\section{Study Design}

This is an observational primary care database study of the comparative effectiveness of treating patients experiencing an asthma exacerbation with a single course of antibiotics alongside oral corticosteroids (OCS) compared to the usual care of OCS alone.

\section{Data Sources and Permissions}

Historical electronic medical records from the Optimum Patient Care Research Database (OPCRD) were used. At the time of this study, the OPCRD contained anonymised, longitudinal medical records for approximately 6 million UK primary care patients, from more than 525 GP practices across the UK. The OPCRD is approved by the Trent Multi-Centre Research Ethics Committee for clinical research use. This study was approved by the Anonymised Data Ethics \& Protocol Transparency committee (ADEPT1519) and registered with the European Network of Centres for Pharmacoepidemiology and Pharmacovigilance (EUPAS 12132). We have followed the STROBE guidance for reporting observational evidence (strobestatement.org). 


\section{Patient population}

Patients were included if they had a prescription for OCS on the same date as a Read code for asthma or wheeze, which was taken to indicate an asthma exacerbation, between 1 January 2004 and 31 December 2014. Index Prescription Date (IPD) was the first date in this study period, when the patient received a prescription for OCS; patients were required to have had no OCS prescriptions (acute or maintenance doses) in the previous 6 months. Patients who received an acute course of OCS were compared to those who received a single acute course of antibiotics in addition to a prescription for OCS at IPD. The first OCS prescription was used so that the IPD represented the start of an exacerbation and not an ongoing exacerbation, and this reduced the chance of previous exacerbation treatment influencing treatment decisions at IPD. Patients were characterised over a 6 -month baseline period immediately prior to IPD and outcomes evaluated in the 12 weeks immediately post IPD (Figure 1).

Inclusion Criteria: aged 2-65 years at IPD; Read codes for asthma (or wheeze if $\leq 5$ years old) on $\geq 3$ occasions ever; $\geq 1$ Read code for asthma (or wheeze if $\leq 5$ years old) during baseline; $\geq 1$ inhaled corticosteroid (ICS) or LTRA prescription during baseline; $\geq 38$ weeks continuous records ( $\geq 26$ weeks prior to IPD and $\geq 12$ weeks following IPD).

Exclusion criteria: received regular antibiotics ( $>5$ prescriptions during baseline); had an additional chronic respiratory condition; aged $\geq 19$ years with a diagnosis of chronic obstructive pulmonary disease (COPD) (Supplementary figure 1).

\section{Outcomes}

The primary study endpoint was time to first primary care consultation coded for asthma/wheeze in the 2week outcome period.

Secondary outcomes were: time to first primary care consultation with a Read code for asthma/wheeze resulting in an OCS prescription with or without antibiotics in the 2-, 6- and 12-week periods post IPD and time to first hospitalisation and emergency department attendance for an exacerbation in the 2-, 6- and 12week periods post IPD.

Exploratory outcomes included the type of antibiotics prescribed at IPD (macrolides versus penicillins), blood eosinophil counts and outcomes in the different paediatric age groups (2-5, 6-12 and 13-18 years).

\section{Statistical analysis}

Data were separated into two age groups: paediatric patients (2-18 year olds) and adults (19-65 year olds). Demographics and clinical characteristics were compared between those given OCS and those given OCS plus antibiotics at IPD, using chi-squared tests. Backward stepwise multivariate logistic regression was used to determine the demographic and clinical characteristics that were predictors of a patient receiving OCS plus antibiotics.

To minimise confounding, individuals from the two groups (OCS plus antibiotics and OCS alone) were matched using 1-1 propensity score matching, using the nearest neighbour method and a caliper width of 0.25. The groups were matched on age, sex, Body Mass Index (BMl; or BMI z-scores in those under 18 year 


\begin{abstract}
old as this gives a measure of relative weight adjusted for child age and sex), GINA category (based on 2018
\end{abstract} guidelines[10]), season of IPD, smoking status, year of IPD and number of consultations for asthma/wheeze in the baseline period. Where matching variables (i.e. smoking status or BMI/zBMI) were missing an additional category for missing values was included; $29.1 \%(1,930 / 6,632)$ of children and $3.7 \%(818 / 22,005)$ of adults had at least one of these two variables missing. The time to primary care consultation for asthma/wheeze and time to primary care consultations for asthma/wheeze resulting in OCS were analysed using Cox proportional hazards regression. The number of patients with at least one primary care consultation and number of those with a respiratory related emergency department visit or hospitalisation were compared using chi-squared or Fisher's exact tests as appropriate. All analyses were performed with R software (www.r-project.org/). R packages used were Hmisc 4.2-0, Gmisc 1.8, htmlTable 1.13.1, survival 2.41-3, ggplot2 3.1.0, survminer 0.4.3.999, Matchlt 3.0.2, forcat 0.4.0, MASS v7.3-47 and the World Health Organisation macros igrowup_standard.r and who2007.r.

\title{
Results
}

28,637 patients fulfilled the eligibility criteria; 22,005 adults (19-65 years) and 6,632 children (2-18 years) (Supplementary figure 1). A large proportion of patients received antibiotics in addition to OCS for the treatment of asthma exacerbations at IPD; 10,012 (45\%) of adults and 2,094 (32\%) of children. There were significant differences in the demographic and clinical characteristics between those who received OCS plus antibiotics compared to those who received OCS alone (Supplementary tables 1-3). The odds of receiving an antibiotic were increased with age, being male, being a smoker or ex-smoker, presenting in winter or in more recent years, while the odds of receiving an antibiotic were decreased in children, those presenting in the summer, those with consultations resulting in a short-acting $\beta$-agonist (SABA) prescription in the previous 6 months or an active rhinitis diagnosis (Table 1).

Following matching, 20,024 (10,012 per group) adults and 4,184 (2,092 per group) children were included in subsequent analyses (Tables 2-3 and supplementary table 4).

\section{Consultations in the 2-, 6- and 12-week outcome period}

The addition of antibiotics to OCS is associated with a reduced risk of having an asthma/wheeze consultation in the following 2 weeks (children HR 0.84 (95\% Cl 0.73-0.96), p=0.012; adults HR 0.86 (95\% Cl 0.81-0.91), $\mathrm{p}<0.001$; Figures $2 a, b, 3)$. In the 2 weeks post-IPD $20.0 \%(2,001 / 10,012)$ of adults who received OCS plus antibiotics had a subsequent asthma/wheeze consultation compared to $22.9 \%(2,289 / 10,012)$ of those who received OCS alone $(p<0.001$, Supplementary figure 2$)$. Similarly, in children $19.6 \%(409 / 2,092)$ receiving OCS plus antibiotics compared to $22.8 \%(478 / 2,092)$ receiving OCS alone had a subsequent consultation within 2 weeks ( $p=0.010$, Supplementary figure 2 ). In the 2 weeks post IPD there was no difference in the time to first asthma/wheeze consultation resulting in a repeated OCS prescription with or without antibiotics, i.e. indicating a new or ongoing exacerbation, for either adults or children (children HR $0.92(95 \% \mathrm{Cl} 0.64-$ 1.33), $p=0.650$; adults HR 1.10 (95\% $\mathrm{Cl} 0.98-1.24), p=0.100)$. When prescription for OCS and/or antibiotics was used as the outcome at 2 weeks post IPD, there was no difference between the groups receiving OCS 
or OCS plus antibiotics prescriptions at IPD in adults, but the risk of a consultation was reduced in children at 2 weeks, but not at 6 or 12 weeks.(2 wk HR 0.69 (95\% Cl 0.50-0.94), p=0.019; supplementary figure 3).

At 6 weeks, the risk of an asthma/wheeze consultation resulting in a repeat OCS prescription with or without antibiotics, was increased in adults who received OCS and antibiotics at IPD compared to OCS alone (HR $1.11(95 \% \mathrm{Cl} 1.01-1.21), \mathrm{p}=0.030$; Figures $2 \mathrm{c}$ and 3). Of the adults who received OCS plus antibiotics at IPD 9.5\% (953/10,012) had a subsequent consultation resulting in an OCS prescription with or without antibiotics compared to $8.6 \%(865 / 10,012)$ who received OCS alone at IPD $(p=0.032$, Supplementary figure 2). However, at 6 weeks in children no significant difference in the risk of an asthma/wheeze consultation resulting in a repeat OCS prescription with or without antibiotics was seen between those who received OCS plus antibiotics at IPD compared to OCS alone at IPD (HR 0.93 (95\% Cl 0.72-1.19), p=0.830; Figures 2d and 3). In the 12-week outcome period there was no difference between the OCS plus antibiotics and OCS alone groups in the time to first for asthma/wheeze consultation for OCS with or without antibiotics, for either adults (HR 1.07 (95\% Cl 0.99-1.15), p=0.090) or children (HR 1.07 (95\% Cl 0.89-1.30), p=0.470). Multivariate Cox proportional hazards regression analysis of the unmatched data produced very similar results for all outcomes.

An exploratory analysis of effect of antibiotics in different paediatric age groups (2-5, 6-12 and 13-18 years) showed similar trends to the group as a whole (data available on request).

An exploratory analysis of adults with low blood eosinophil counts (0-0.2 10\%/L) compared to high blood eosinophil counts (>0.2 10\%/L) was conducted. The addition of antibiotics at IPD was significantly associated with a reduced risk of an asthma/wheeze consultation in the 2 weeks post IPD, which was of a similar magnitude in both those with high and with low blood eosinophil counts (High eos HR 0.87 (95\% Cl $0.77-$ 0.98), p=0.018; Low eos HR $0.84(95 \% \mathrm{Cl}$ 0.75-0.94), p=0.003; Supplementary figure 4)). In both those with a high blood eosinophil count and a low blood eosinophil count there was no difference between the OCS and OCS plus antibiotic groups in the time to first asthma/wheeze consultation for OCS with or without antibiotics in the 2, 6 and 12 week outcome periods.

\section{Emergency department attendances and hospitalisations}

Only a small number of patients experienced a severe exacerbation, defined as requiring an emergency department attendance or hospitalisation ( $<0.5 \%$ of patients had an emergency department attendance or hospitalisation in the 12 weeks post IPD) so Cox proportional hazards regression was not performed. There were no significant differences between the OCS plus antibiotics and OCS alone groups in the number of patients with an emergency department attendance or hospitalisation (Table 4).

\section{Antibiotic type: Penicillins versus Macrolides}

In children given antibiotics at IPD, 86.1\% (1,802/2,092) received penicillins and 10.0\% $(210 / 2,092)$ received macrolides. Of those who received OCS plus penicillin, $19.0 \%$ had an asthma/wheeze consultation in the 2 
weeks post IPD, which was significantly less than in those who received OCS alone $(22.8 \%, p=0.004)$. However, in those given macrolides the percentage of children with an asthma/wheeze consultation in the first 2 weeks was not significantly different $(23.8 \%, p=0.82$, Figure $4 a)$ compared to OCS alone.

In the adults who received antibiotics at IPD $73.6 \%(7,371 / 10,012)$ received penicillins and $17.1 \%$ $(1,708 / 10,012)$ received macrolides. Similarly to in children, penicillins, but not macrolides, at IPD were associated with a significant reduction in the number of patients having a subsequent asthma/wheeze consultation in the 2 weeks post IPD compared to OCS alone (penicillins $19.1 \%$ vs $22.9 \%$ OCS alone, $p<0.001$; macrolides $21.8 \%$ vs $22.9 \%$ OCS alone, $p=0.37$, Figure $4 b$ ).

In both the paediatric and adult groups neither penicillins nor macrolides were associated with a significant difference in the number of patients having an asthma/wheeze consultation resulting in an OCS prescription with or without an antibiotic, in the 2- or 6-week outcome periods (children 2-week outcome $p=0.33$, 6-week outcome $p=0.68$; adults 2-week outcome $p=0.29$, 6-week outcome $p=0.16$; Figure 4a\&b).

\section{Discussion}

We have investigated the effectiveness of adding antibiotics alongside OCS for the treatment of asthma exacerbations in a heterogeneous real-life population comprising both adult and paediatric asthma patients. The addition of antibiotics to OCS is associated with a small reduction in the absolute risk of a subsequent asthma/wheeze consultation in the following 2 weeks; around $3 \%$ fewer patients having consultations for asthma/wheeze. However, there was no difference in the rates of prescription of OCS and/or antibiotics at 2 weeks. One possible explanation for this is that GPs used a different read code at follow up at 2 weeks when further antibiotic treatment was prescribed. In contrast, in adults, but not children, there was a slightly increased risk of a consultation for a new/ongoing exacerbation (defined as a repeated OCS prescription) in the 6 weeks post IPD. The very low numbers of emergency department attendances and hospitalisations, which may be due partly to the poor recording of emergency department attendances and hospitalisations in primary care databases, make it difficult to draw firm conclusions. However, we saw no difference in the numbers of emergency department attendances or hospitalisations associated with the addition of antibiotics. While there were statistically significant differences, the magnitude was relatively small, and needs to be balanced against the adverse effects of antibiotic use, both at individual and at community level. The lack of impact on repeat prescription of OCS and/or antibiotics suggests that addition of antibiotics does not reduce treatment failure and thus healthcare resource utilisation. Our analysis occurred at group aggregated level, hence it is possible that while for most patients the addition of an antibiotic is of no benefit, there may be subgroups who benefit, and this should be a focus of further research. In a post hoc analysis looking at blood eosinophil levels we found no significant differences in the any of the outcomes between those with high blood eosinophil levels ( $\left.>0.2 \times 10^{9} / L\right)$ and those with low blood eosinophil counts. In a primary care population, the routine addition of antibiotics appears to be of minimal, if any, clinical benefit in treating asthma exacerbations, especially when considering the major risk of antibiotic resistance associated with antibiotic overuse [11].

The small increase in time until a subsequent asthma/wheeze consultation in patients prescribed antibiotics may be partly explained by patients receiving antibiotics feeling that their expectations have been met, making them less likely to return for further treatment for ongoing symptoms. A course of antibiotics will likely 
last for 5-7 days, compared to the usual shorter course of OCS, so it could be expected that patients prescribed antibiotics who have ongoing symptoms are going to finish the longer course of antibiotics, before returning for a subsequent consultation. A limitation of this study is that we do not have information regarding delayed prescribing, as this is not well recorded in primary care databases. A previous study in UK primary care has suggested around $18 \%$ of antibiotic prescribing for lower respiratory tract infections (LRTI) in adults may be delayed prescribing, where patients are advised to take one treatment first followed by the second if symptoms are unresolved [12]. Therefore, in patients who received both OCS and antibiotics at IPD the time until those who have ongoing symptoms return for a subsequent consultation could be extended, biasing the primary outcome to favour OCS and antibiotics at IPD. While antibiotics may reduce the chances of patients returning with a LRTI, those with LRTIs are at increased risk of having an exacerbation [13]. This may in part explain why we observed an increased risk of exacerbations at 6 weeks in the antibiotic treated adult population. Although we matched our patient groups for a number of variables there is the potential for residual confounding. The higher number of co-morbidities in the adult population receiving OCS plus antibiotics may have influenced the prescribing at 2 and 6 weeks if symptoms had not fully resolved. There may have been other factors, such as positive sputum cultures, that guided treatment decisions which are not well recorded within the database. Time to the first primary care consultation for asthma/wheeze was only analysed at 2 weeks post IPD; this outcome included all consultations with an asthma or wheeze Read code. It was felt patients returning within 2 weeks most likely represent those with ongoing exacerbations rather than routine/follow-up appointments. A further limitation is that we required an asthma/wheeze Read code at follow up, however, analysis of a very small random subset $(0.1 \%$ of the sample size) suggests we have missed at least $7.5 \%$ of respiratory related consultations at 2 weeks post IPD, as other Read codes (e.g. for chest infection) were used.

Despite some RCTs suggesting a beneficial effect of macrolide antibiotics in both treating and preventing exacerbations, $[6,7,14]$ there are a number of studies that have found no benefit in the use of antibiotics in adults receiving hospital treatment for asthma exacerbations. A retrospective cohort study of adult asthmatics hospitalised for asthma exacerbations found an increase in the length of hospital stay in those prescribed antibiotics.[15] A RCT of adult asthmatics hospitalised with asthma exacerbations found amoxicillin compared to placebo had no significant effect on length of hospital stay, symptoms or lung function.[16] Similarly, azithromycin compared to placebo had no significant effect on quality-of-life questionnaire scores, lung function and symptom score in adult asthmatics presenting with asthma exacerbations in secondary care.[17]

Our study benefits from a large heterogeneous real-life population that includes both paediatric and adult patients and addresses an important need in assessing antibiotic use in asthma exacerbations, as highlighted by a recent Cochrane review.[9] The mixed population of patients included represent the asthmatic population typically seen in primary care, where most asthma exacerbations are treated, and where it can be difficult to separate what is a non-infective asthma exacerbation and what is a (mostly viral) infection. It can be difficult to distinguish between a non-infective asthma exacerbation and LRTI as the symptoms are often indistinguishable, particularly, but not exclusively, in those with a previous history of asthma.[18] Furthermore, exacerbations and infections are not independent events; respiratory infections are a major trigger of asthma exacerbations.[19] However, viral infections are thought to trigger up to $85 \%$ of acute 
We found high levels of antibiotic prescribing, which is perhaps surprising given the addition of antibiotics is currently not recommended within the guidelines for the treatment of asthma exacerbations.[4] Antibiotics may be prescribed due to the uncertainties around the definition and symptoms of asthma exacerbations and there being multiple potential causes of the increased respiratory symptoms, for some of which antibiotics may be beneficial. It is possible some of the antibiotic prescribing at IPD could be for co-morbidities; as this is a real-life population some patients may have presented with other infections, for example otitis media, that prompted the antibiotic prescription, alongside symptoms of an asthma exacerbation. Information on such comorbidities was not collected, but many of the other potential diagnoses/infections would likely be of viral origin. The level of antibiotic prescribing observed here was similar to that reported in previous studies. A 1992/1993 study found that approximately $40 \%$ of asthmatic patients experiencing an exacerbation managed in UK primary care, were given antibiotics.[21] In another study $44.6 \%$ of adult asthmatics seeking emergency treatment for an asthma exacerbation had received antibiotics in the previous 4 weeks.[17] Antibiotic prescribing was more common in certain groups: older people, males, smokers or ex-smokers, and was more common in winter, and interestingly increased between 2004 and 2014. The increase in antibiotic prescribing could be due to increased time pressures, reduced access to GP appointments over this period, related to increased concern about the consequences of missing something or not meeting increased patient/carer expectations [22,23,24].

Patients prescribed penicillins alongside OCS had a small reduction in the odds of a subsequent asthma/wheeze consultation compared to OCS alone. This is consistent with a previous study of penicillin use in asthma[8] and studies that have found penicillin treatment for COPD exacerbations, and for LRTIs in patients without respiratory disease, is associated with a lower risk of needing repeat antibiotics.[20,21] In those prescribed macrolides alongside OCS the odds of a subsequent asthma/wheeze consultation were not significantly different compared to those receiving OCS alone. Hence the observed statistically significant benefit was associated with only penicillins, not macrolides. This apparent benefit with penicillins could be an artefact of the GPs choosing to prescribe macrolides to those with more severe illness that they may have felt would not be adequately treated with penicillins. This could explain the divergence with previous RCTs that found beneficial effects of macrolides[6,7], although it should be noted it is difficult to draw firm conclusions from our study given the number of patients prescribed macrolides is relatively low. The patients in our study and in other studies where the beneficial effect of penicillins have been seen[8,25,26] have presented in primary care, whereas the studies showing macrolide benefits have been in patients that have presented in the emergency department.[6,7]. Patients attending the emergency department may have different underlying disease severity or a different microbiome that makes macrolides more effective in that scenario. 
In conclusion, we found antibiotic use to be common in asthma exacerbations but did not find clear evidence of a clinically significant benefit of the addition of antibiotics to usual care.

\section{Acknowledgements}

This project was supported by the Respiratory Effectiveness Group. Data and data management support was provided in-kind by Optimum Patient Care (www.opcrd.co.uk) and Derek Skinner at OPC. Clare Murray is supported by the NIHR Manchester Biomedical Research Centre.

\section{References}

1. Korppi M. Management of bacterial infections in children with asthma. Expert Rev Anti Infect Ther. 2009; 7:869-77.

2. Nicholson KG, Kent J, Ireland DC. Respiratory viruses and exacerbations of asthma in adults. BMJ. 1993; 307: 982-986.

3. Johnston SL. Macrolide antibiotics and asthma treatment. J Allergy Clin Immunol. 2006; 117:1233-6.

4. Global Initiative for Asthma (GINA). Pocket Guide for Asthma Management and Prevention. Updated 2019. Available online at: https://ginasthma.org/wp-content/uploads/2019/04/GINA-2019-main-PocketGuide-wms.pdf (last accessed: 16/09/19)

5. Reddel, HK, Taylor DR, Bateman ED, Boulet L-P, Boushey HA, Busse WW, Casale TB, Chanez P, Enright PL, Gibson PG, de Jongste JC, Kerstjens HAM, Lazarus SC, Levy ML, O’Byrne PM, Partridge MR, Pavord ID, Sears MR, Sterk PJ, Stoloff SW, Sullivan SD, Szefler SJ, Thomas MD, \& Wenzel SE, on behalf of the American Thoracic Society/European Respiratory Society Task Force on Asthma Control and Exacerbations. An official American Thoracic Society/European Respiratory Society statement: asthma control and exacerbations: standardizing endpoints for clinical asthma trials and clinical practice. Am J Respir Crit Care Med. 2009; 180:59-99, 2009.

6. Johnston SL, Blasi F, Black PN, Martin RJ, Farrell DJ, Nieman RB; TELICAST Investigators. The effect of telithromycin in acute exacerbations of asthma. N Engl J Med. 2006; 354:1589-600.

7. Koutsoubari I, Papaevangelou V, Konstantinou GN, Makrinioti H, Xepapadaki P, Kafetzis D, Papadopoulos NG. Effect of clarithromycin on acute asthma exacerbations in children: an open randomized study. Pediatr Allergy Immunol. 2012; 23:385-90.

8. Stolbrink M, Bonnett LJ, Blakey JD. Antibiotic Choice and Duration Associate with Repeat Prescriptions in Infective Asthma Exacerbations. J Allergy Clin Immunol Pract. 2019; 7:548 - 553.

9. Normansell, R., Sayer, B., Waterson, S., Dennett, E.J., Del Forno, M., and Dunleavy, A. Antibiotics for exacerbations of asthma. Cochrane Database Syst Rev. 2018; 6: CD002741.

10. Global Initiative for Asthma (GINA). Pocket Guide for Asthma Management and Prevention. Updated 2018. Available online at: https://ginasthma.org/wp-content/uploads/2018/03/wms-GINA-main-pocketguide 2018-v1.0.pdf (last accessed: 8/3/2020)

11. World Health Organization. Antimicrobial resistance - fact sheet. 2018 https://www.who.int/newsroom/fact-sheets/detail/antimicrobial-resistance (last accessed: 19/5/2020)

12. Little P, Stuart B, Smith S, Thompson MJ, Knox K, van den Bruel A et al. Antibiotic prescription strategies and adverse outcome for uncomplicated lower respiratory tract infections: prospective cough complication cohort (3C) study BMJ 2017; 357 :j2148

13. Price D, Wilson AM, Chisholm, A, Rigazio A, Burden A, Thomas M, King C. Predicting frequent asthma exacerbations using blood eosinophil count and other patient data routinely available in clinical practice. Journal of Asthma and Allergy. 2016; 9:1-12.

14. Gibson PG, Yang IA, Upham JW, Reynolds PN, Hodge S, James AL, Jenkins C, Peters MJ, Marks GB, Baraket M, Powell H, Taylor SL, Leong LEX, Rogers GB \& Simpson JL. Effect of azithromycin on asthma exacerbations and quality of life in adults with persistent uncontrolled asthma (AMAZES): a randomised, double-blind, placebo-controlled trial. The Lancet. 2017; 390:659-668.

15. Stefan MS, Shieh MS, Spitzer KA, Pekow PS, Krishnan, JA, \& Au DH, Lindenauer PK. Association of Antibiotic Treatment With Outcomes in Patients Hospitalized for an Asthma Exacerbation Treated With Systemic Corticosteroids. JAMA Internal Medicine. 2019;179(3):333-339. 
16. Graham VAL, Knowles GK, Milton AF, Davies, RJ. Routine Antibiotics in Hospital Management of Acute Asthma. The Lancet. 1982; 319:418-421.

17. Johnston, SL, Szigeti M, Cross M, Brightling C, Chaudhuri R, Harrison T, Mansur A, Robison L, Sattar Z, Jackson D, Mallia P, Wong E, Corrigan C, Higgins B, Ind P Singh D, Thomson NC, Ashby D, Chauhan A; For the AZALEA Trial Team Azithromycin for Acute Exacerbations of Asthma: The AZALEA Randomized Clinical Trial. JAMA Intern Med. 2016; 176:1630-1637.

18. Guibas GV, Tsolia M, Christodoulou I, Stripeli F, Sakkou Z, Papadopoulos NG. Distinction between rhinovirus-induced acute asthma and asthma-augmented influenza infection. Clin Exp Allergy. 2018; 48(5):536-543.

19. Xepapdaki P, Megremis S, Kitsioulis NA, Papadopoulos NG. Infections in the nose and exacerbations of chronic respiratory disorders. In: Bachert C, Bourdin A, Chanez P, eds. The Nose and Sinuses in Respiratory Disorders (ERS Monograph). Shefield, European Respiratory Society, 2017; pp000-000.

20. Saraya T, Kurai D, Ishii H, Ito A, Sasaki Y, Niwa S, Kiyota N, Tsukagoshi H, Kozawa K, Goto $\mathrm{H}$, Takizawa $\mathrm{H}$. Epidemiology of virus-induced asthma exacerbations: with special reference to the role of human rhinovirus. Front Microbiol. 2014; 5: 226.

21. Neville RG, Hoskins G, Smith B, Clark RA. How general practitioners manage acute asthma attacks. Thorax. 1997; 52:153-156.

22. Ashworth $M$, White $P$, Jongsma $H$, Schofield $P$, Armstrong $D$. Antibiotic prescribing and patient satisfaction in primary care in England: cross-sectional analysis of national patient survey data and prescribing data. Br J Gen Pract. 2016;66(642):e40-e46. doi:10.3399/bjgp15X688105

23. SernaMC, Real J, Ribes $E$ et al. Factors determining antibiotic prescription in primary care. Enferm Infecc Microbiol Clin 2011; 29: 193-200.

24. Lucas PJ, Cabral C, Hay AD, Horwood J. A systematic review of parent and clinician views and perceptions that influence prescribing decisions in relation to acute childhood infections in primary care. Scand J Prim Health Care. 2015;33(1):11-20. doi:10.3109/02813432.2015.1001942

25. Stolbrink M, Bonnett LJ, Blakey JD. Antibiotics for COPD exacerbations: does drug or duration matter? A primary care database analysis. BMJ Open Resp Res. 2019; 6:e000458.

26. Stolbrink M, Bonnett LJ, Blakey JD. Amoxicillin is associated with a lower risk of further antibiotic prescriptions for lower respiratory tract infections in primary care - A database analysis spanning over 30 years. Eur Clin Respir J. 2018; 5(1): 1529535. 


\section{Tables}

Table 1. Predictors of receiving Oral Corticosteroids plus antibiotics at Index Prescription Date (IPD).

\begin{tabular}{lrrr}
\hline & Odds ratio & $\mathbf{9 5 \%}$ Cl & p-value \\
\hline Age & & & \\
$2-5$ yrs & 0.80 & $(0.67-0.95)$ & 0.0126 \\
$6-12$ yrs & 0.75 & $(0.66-0.85)$ & $<0.0001$ \\
$13-18$ yrs & 0.91 & $(0.80-1.04)$ & 0.1526 \\
$19-25$ yrs & REF & & \\
$26-35$ yrs & 1.07 & $(0.96-1.20)$ & 0.2305 \\
$36-45$ yrs & 1.18 & $(1.06-1.31)$ & 0.0026 \\
$46-55$ yrs & 1.38 & $(1.24-1.54)$ & $<0.0001$ \\
$56-65$ yrs & 1.62 & $(1.45-1.80)$ & $<0.0001$ \\
Male & 1.10 & $(1.04-1.15)$ & $<0.0003$ \\
Current Smoker & 1.56 & $(1.46-1.67)$ & $<0.0001$ \\
Ex-Smoker & 1.09 & $(1.03-1.17)$ & 0.0051 \\
Obese & 1.06 & $(1.00-1.13)$ & 0.0500 \\
Summer IPD & 0.82 & $(0.76-0.88)$ & $<0.0001$ \\
Autumn IPD & 1.08 & $(1.01-1.16)$ & 0.0210 \\
Winter IPD & 1.26 & $(1.18-1.35)$ & $<0.0001$ \\
IPD 2004-2007 & REF & & \\
IPD 2007-2009 & 1.18 & $(1.11-1.25)$ & $<0.0001$ \\
IPD 2010-2012 & 1.42 & $(1.33-1.51)$ & $<0.0001$ \\
IPD 2013-2014 & 1.55 & $(1.43-1.69)$ & $<0.0001$ \\
1 SABA consult in baseline & 0.95 & $(0.90-1.00)$ & 0.0373 \\
2 SABA consults in baseline & 0.88 & $(0.81-0.95)$ & 0.0019 \\
Active rhinitis & 0.90 & $(0.84-0.96)$ & 0.0025 \\
\hline SABA: Short-Acting Beta Agonist & & &
\end{tabular}

SABA: Short-Acting Beta Agonist 
Table 2. Demographic and clinical characteristics for 2-18 year olds, following propensity score matching. Values are $\mathrm{n}(\%)$.

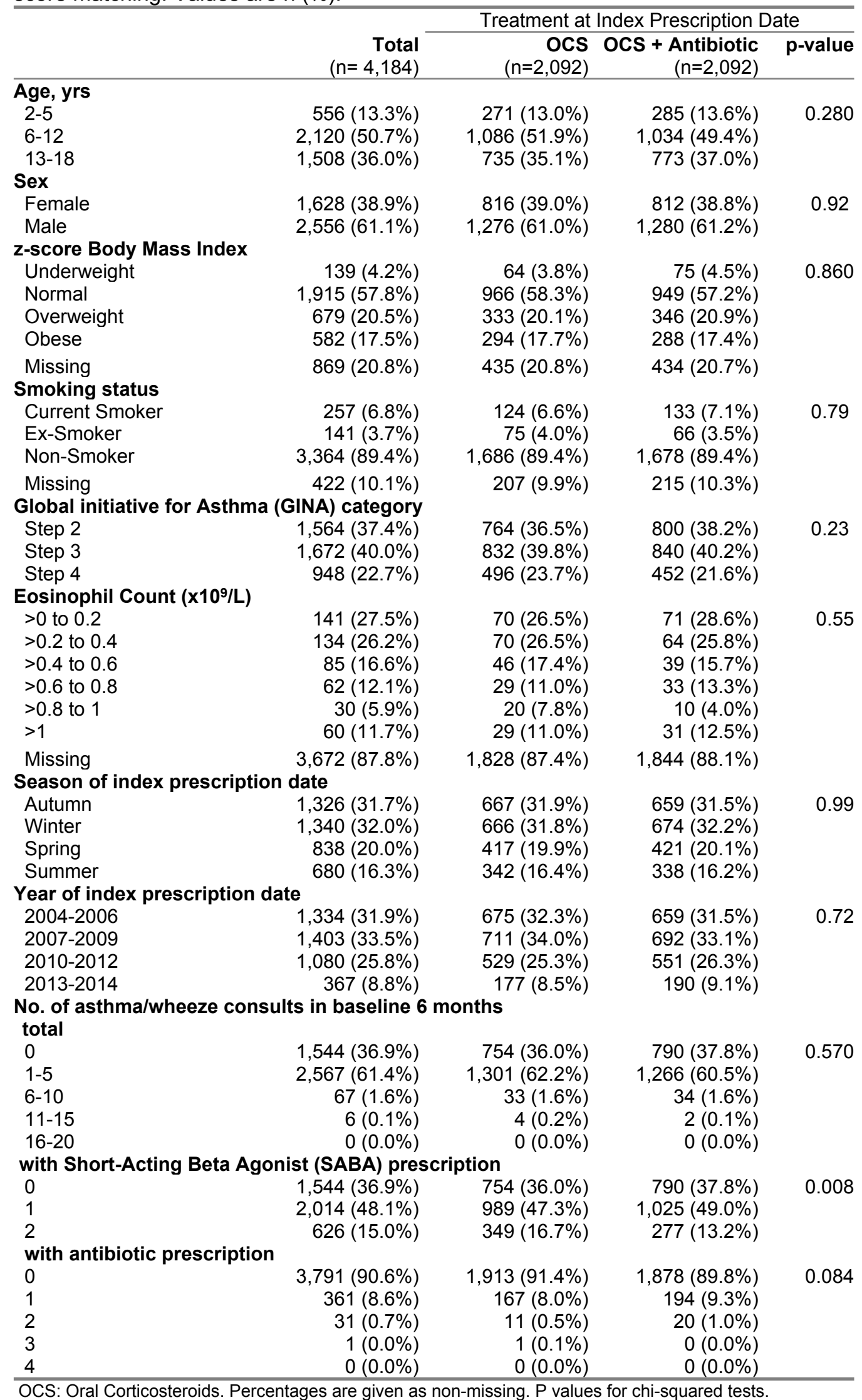


Table 3. Demographic and clinical characteristics for 19-65 year olds, following propensity score matching. Values are $\mathrm{n}(\%)$.

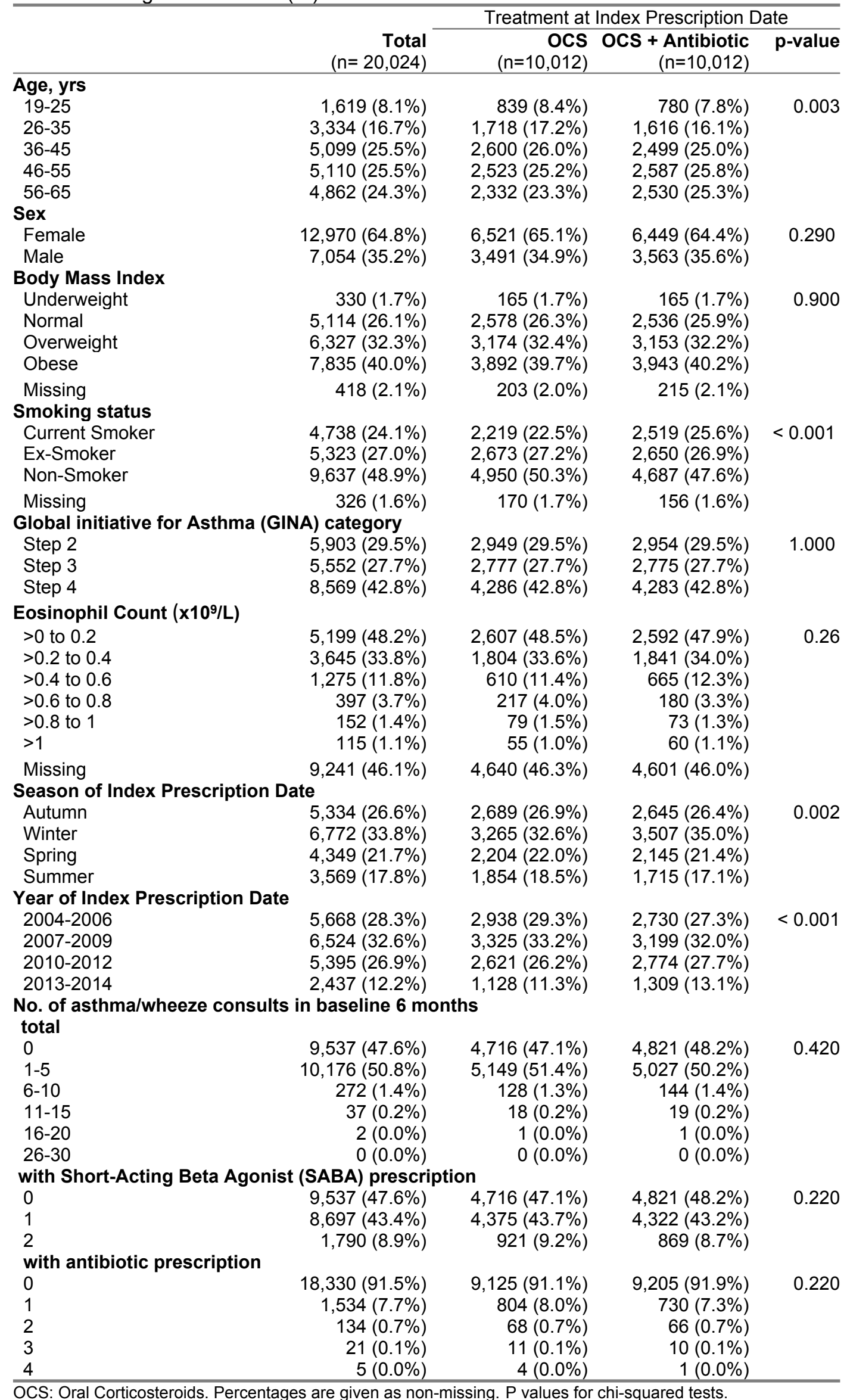


Table 4. Number of patients with at least one severe exacerbation

\begin{tabular}{|c|c|c|c|c|c|c|c|}
\hline \multirow[b]{2}{*}{$\begin{array}{l}\text { Outcome } \\
\text { period }\end{array}$} & & \multicolumn{3}{|c|}{$2-18$ year olds } & \multicolumn{3}{|c|}{$19-65$ year olds } \\
\hline & & $\begin{array}{r}\text { OCS } \\
(n=2,092)\end{array}$ & $\begin{array}{r}\text { OCS + } \\
\text { Antibiotic } \\
(n=2,092)\end{array}$ & p-value & $\begin{array}{r}\text { OCS } \\
(n=10,012) \\
\end{array}$ & $\begin{array}{r}\text { OCS + } \\
\text { Antibiotic } \\
(n=10,012)\end{array}$ & -value \\
\hline \multirow[t]{2}{*}{2 weeks } & Emergency department visit & $4(0.2 \%)$ & $2(0.1 \%)$ & 0.69 & $20(0.2 \%)$ & $22(0.2 \%)$ & 0.88 \\
\hline & Hospitalisation & $3(0.1 \%)$ & $5(0.2 \%)$ & 0.73 & $22(0.2 \%)$ & $24(0.2 \%)$ & 0.88 \\
\hline \multirow[t]{2}{*}{6 weeks } & Emergency department visit & $7(0.3 \%)$ & $5(0.2 \%)$ & 0.77 & $33(0.3 \%)$ & $37(0.4 \%)$ & 0.72 \\
\hline & Hospitalisation & $9(0.4 \%)$ & $6(0.3 \%)$ & 0.61 & $35(0.3 \%)$ & $31(0.3 \%)$ & 0.71 \\
\hline \multirow[t]{2}{*}{12 weeks } & Emergency department visit & $11(0.5 \%)$ & $9(0.4 \%)$ & 0.82 & $51(0.5 \%)$ & $54(0.5 \%)$ & 0.84 \\
\hline & Hospitalisation & $12(0.6 \%)$ & $7(0.3 \%)$ & 0.36 & $44(0.4 \%)$ & $48(0.5 \%)$ & 0.75 \\
\hline
\end{tabular}


Figure 1. Study Schematic

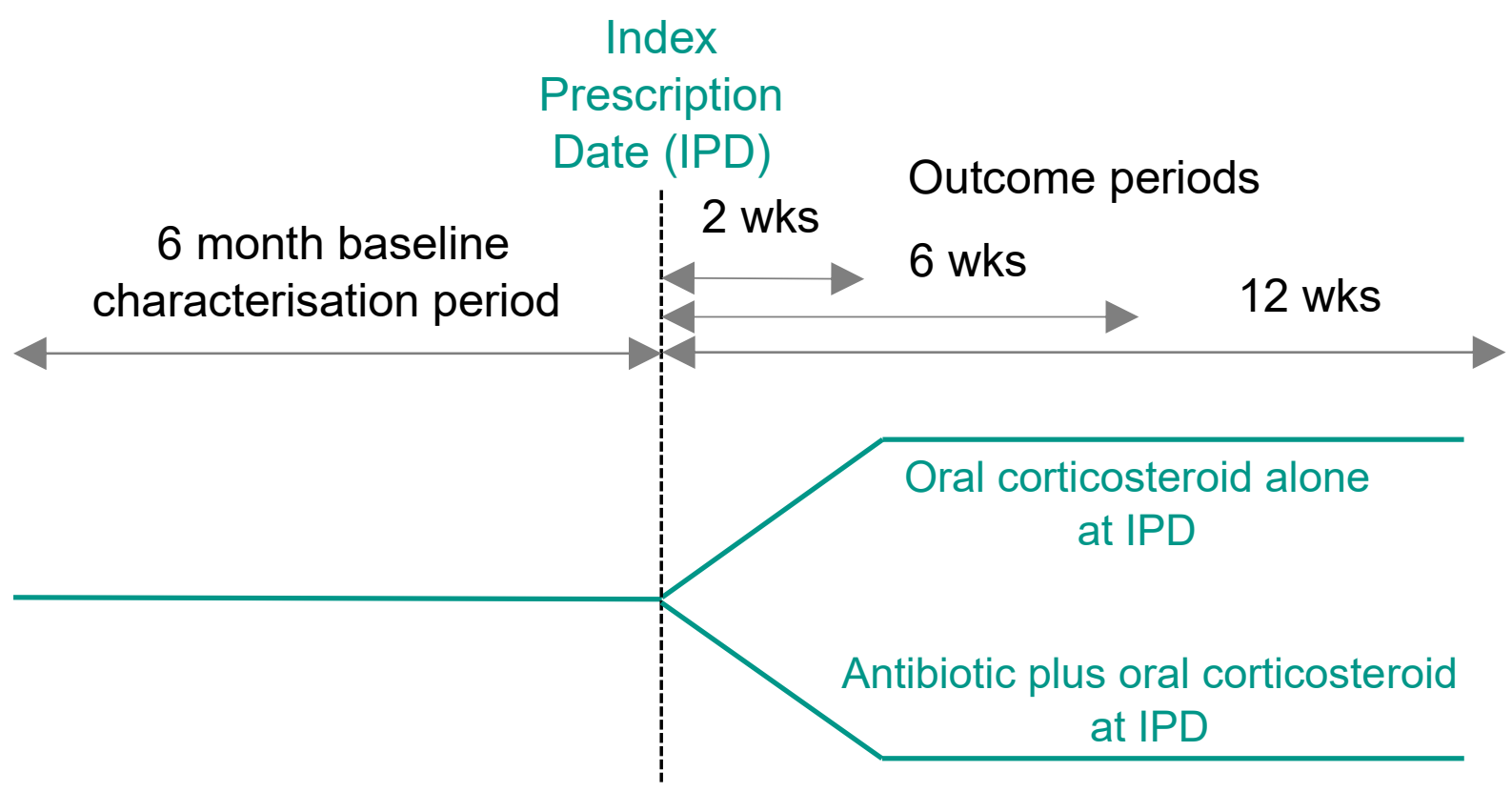


Figure 2. Survival analysis of time to first consultation.

a) Time to first asthma/wheeze consult in $2 \mathrm{wk}$ outcome period for 2-18 year olds

b) Time to first asthma/wheeze consult in $2 \mathrm{wk}$ outcome period for 19-65 year olds

HR $0.84(95 \%$ Cl 0.73-0.96)
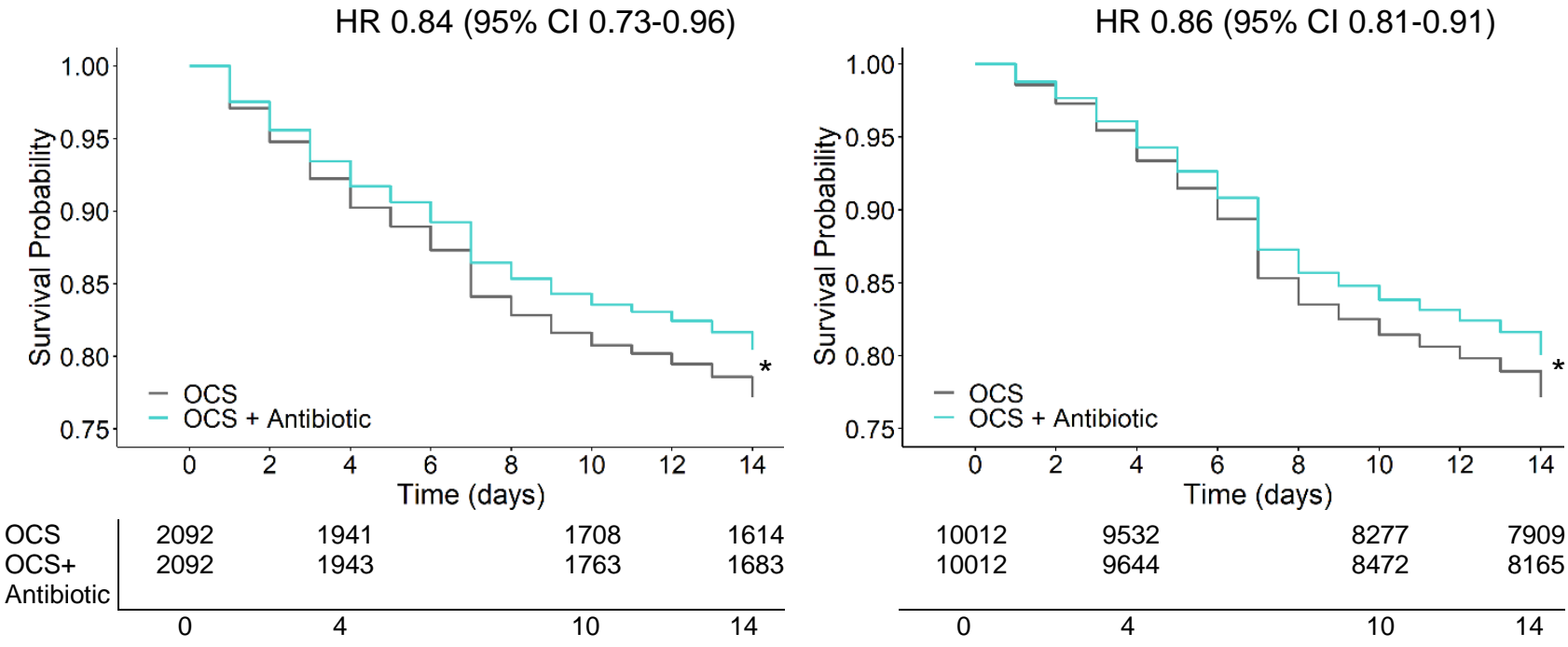

c) Time to first asthma/wheeze consult for OCS with/without antibiotic in 6 wk outcome period for 2-18 year olds

HR 0.93 (95\% Cl 0.72-1.19)

d) Time to first asthma/wheeze consult for OCS with/without antibiotics in 6 wk outcome period for 19-65 year olds
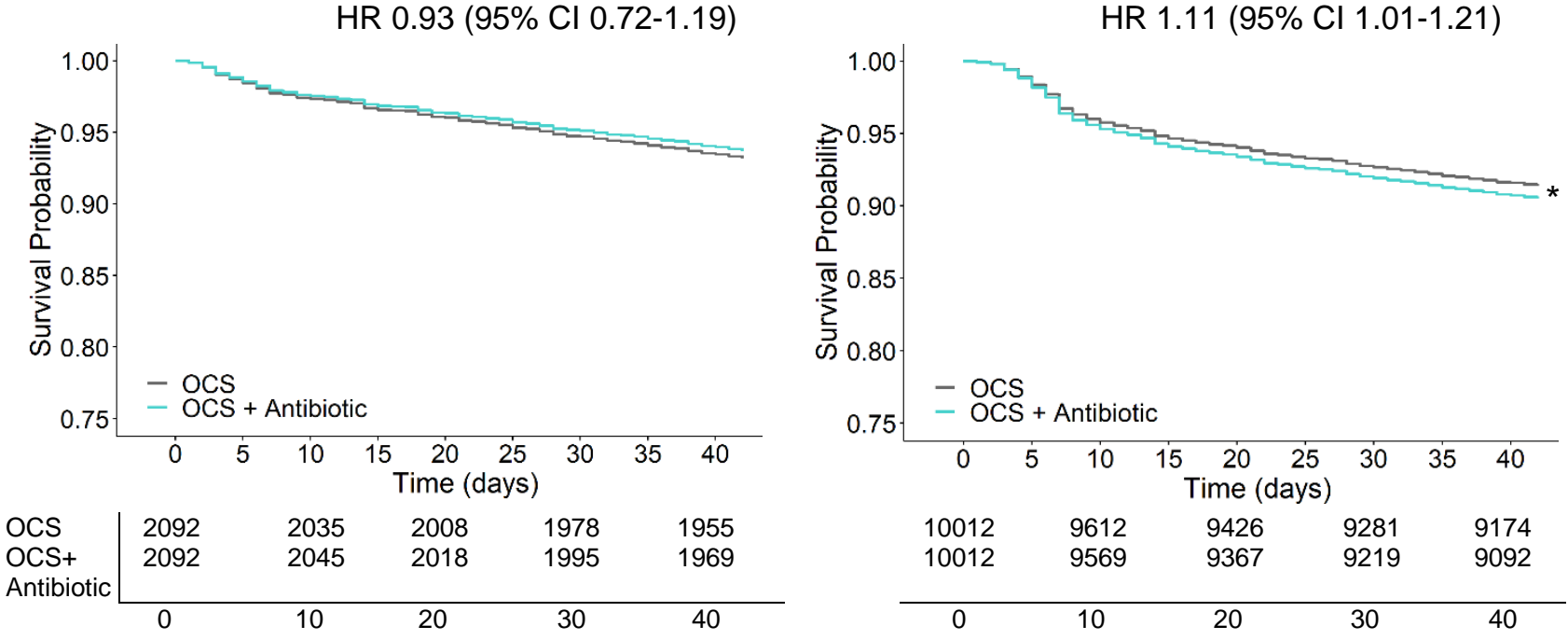

OCS: Oral Corticosteroids. ${ }^{\star} \mathrm{p}<0.05$. 
Figure 3. Hazard ratios $(95 \% \mathrm{Cl})$ for oral corticosteroids (OCS) plus antibiotics compared to oral corticosteroids alone.

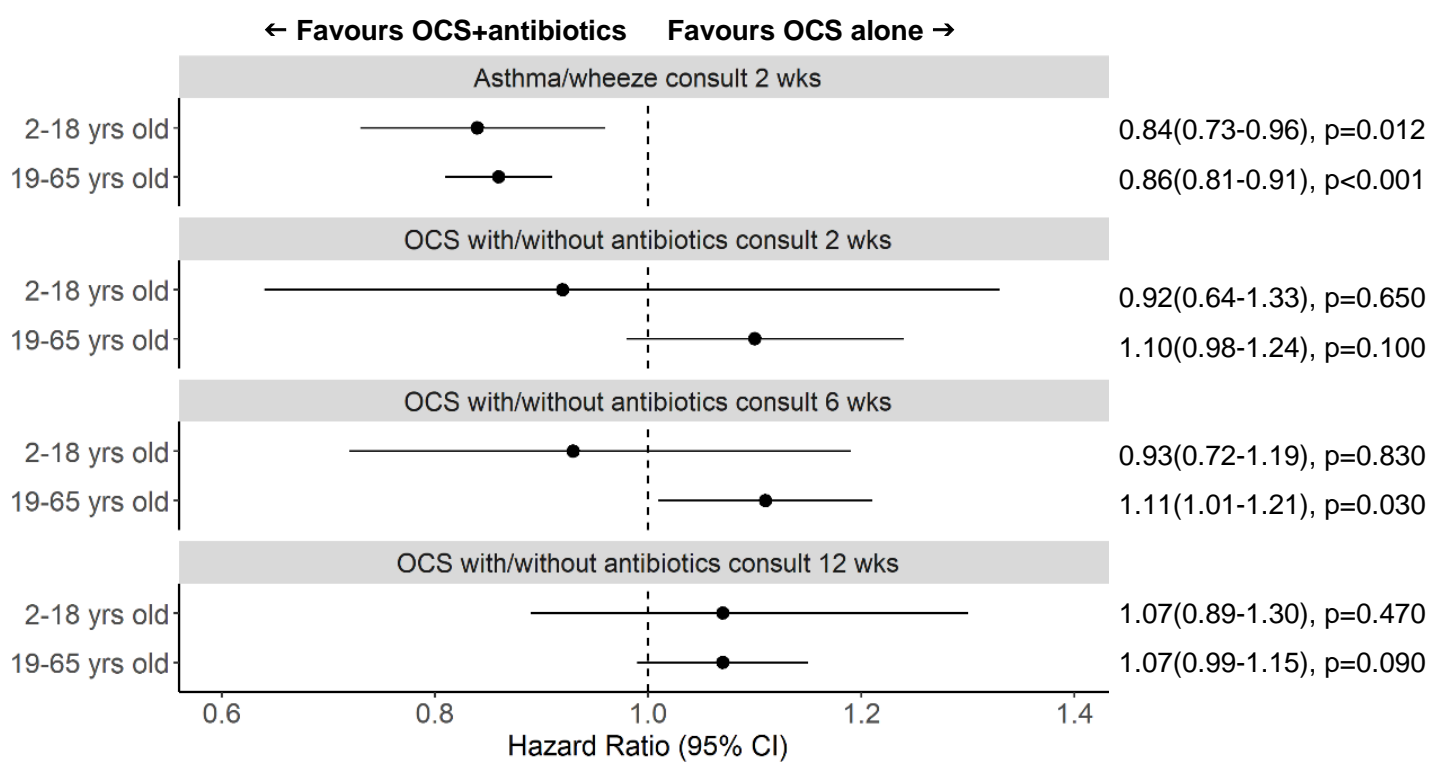


Figure 4. Comparison of the effectiveness of penicillins versus macrolides.

a) Percentage of 2-18 yr olds with at least one primary care consultation by treatment type at IPD (2,092 received OCS alone, 1,802 received OCS+penicillins and 210 received OCS+macrolides)

Asthma/wheeze consult in 2 weeks post IPD

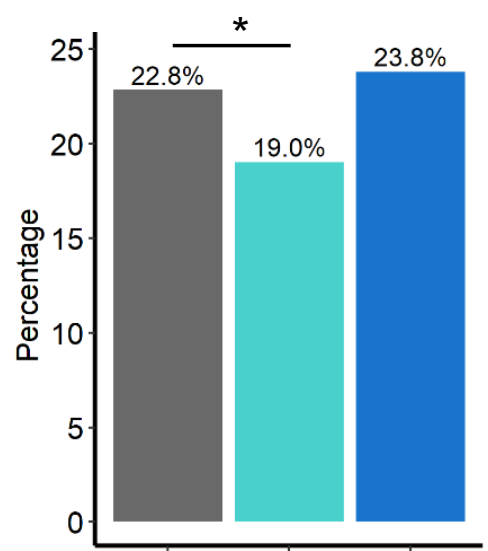

No. of patients with consult
OCS with/without Antibiotics consult in 2 weeks post IPD

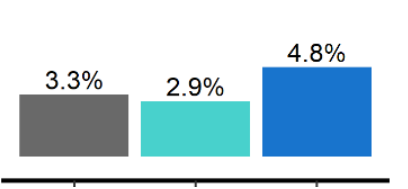

69

\section{OCS with/without Antibiotics consult \\ in 6 weeks post IPD}

OCS alone OCS+Penicillins

OCS+Macrolides

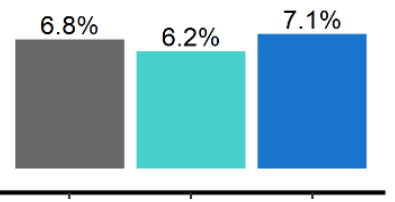

$143 \quad 112 \quad 15$

b) Percentage of 19-65 yr olds with at least one primary care consultation by treatment type at IPD (10,012 received OCS alone, 7,371 received OCS+penicillins and 1,708 received OCS+macrolides)

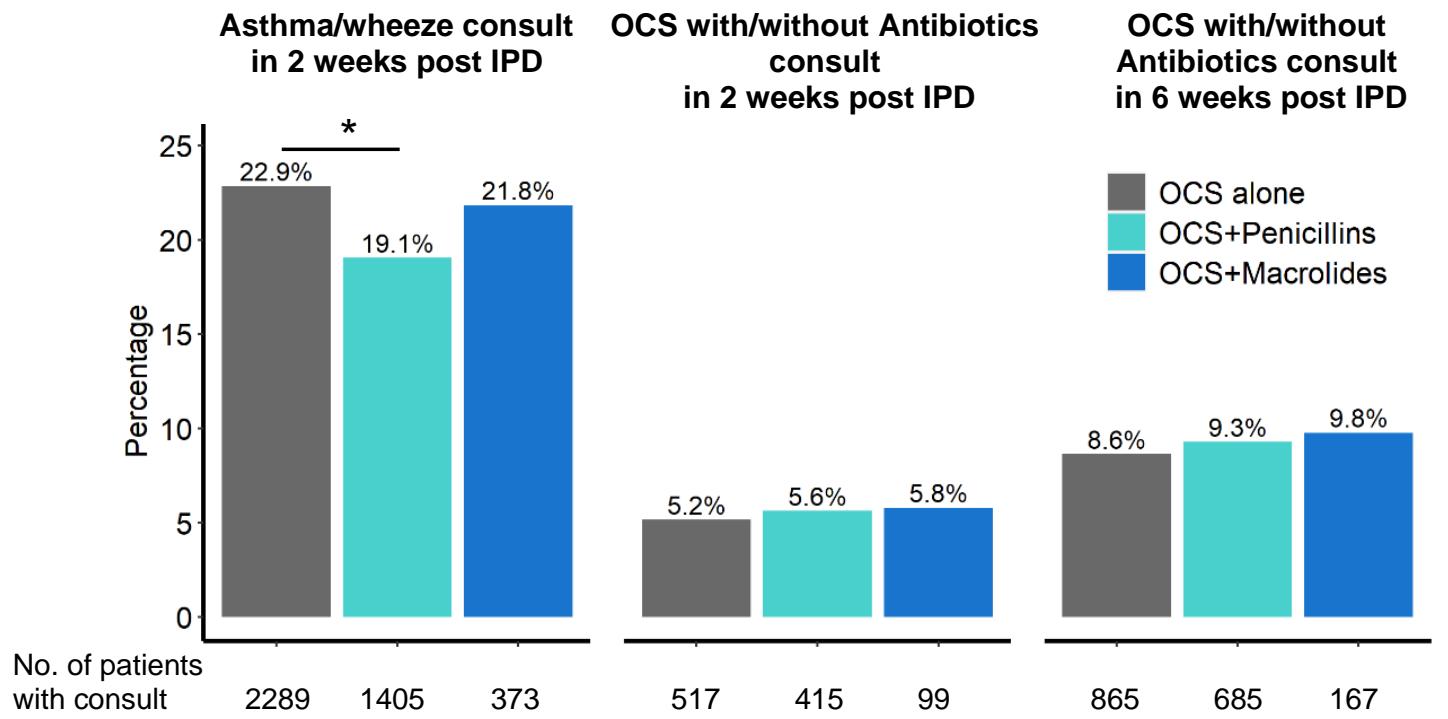

OCS: Oral Corticosteroids; IPD: Index Prescription Date. ${ }^{\star} \mathrm{p}<0.05$ 
Supplementary figure 1. Patient flow

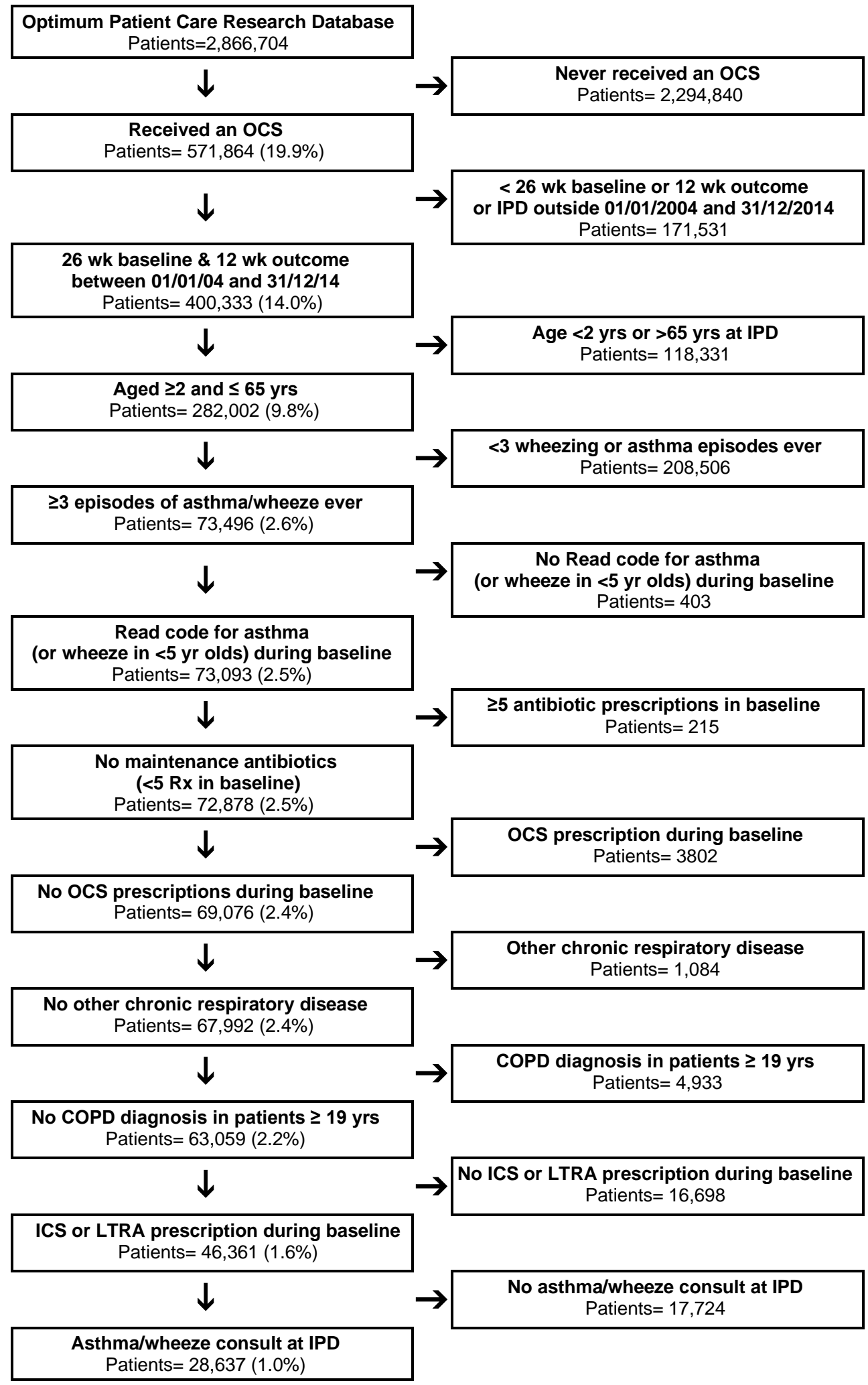

OCS: Oral Corticosteroid; IPD: Index Prescription Date; COPD: Chronic Obstructive Pulmonary Disease; ICS: Inhaled Corticosteroid; LTRA: Leukotriene Receptor Antagonist. 
Supplementary figure 2. Percentage of 2-18 year olds ( $n=2092$ per group) and 19-65 year olds ( $n=10012$ per group) with at least one primary care consultation

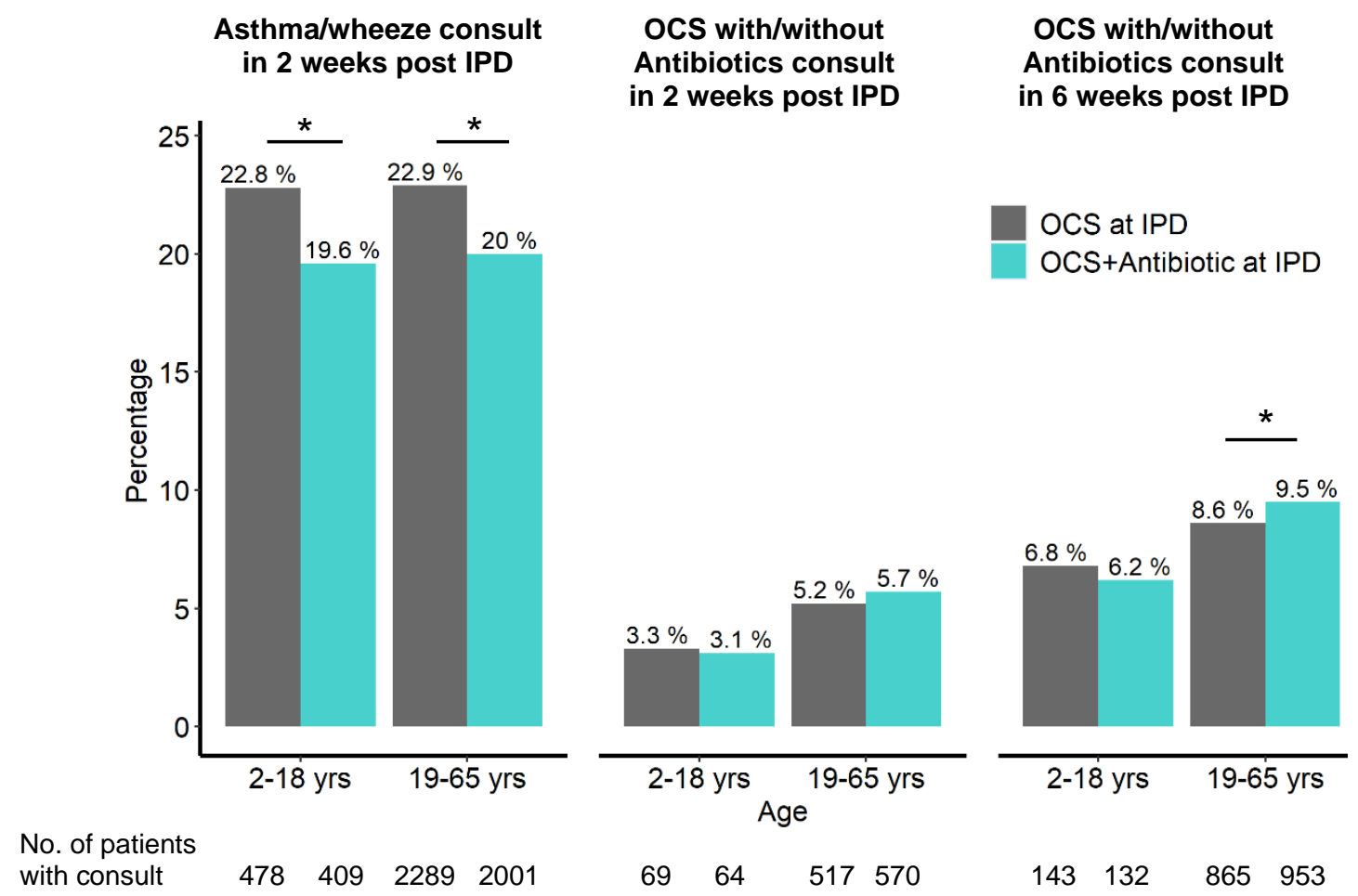

OCS: Oral Corticosteroids; IPD: Index Prescription Date. ${ }^{*} p<0.05$ 
Supplementary figure 3 . Hazard ratios $(95 \% \mathrm{Cl}$ ) for oral corticosteroids (OCS) plus antibiotics compared to oral corticosteroids alone using time to OCS and/or antibiotics as the outcome measure.

a) In $19-65$ yr olds $(n=20,024)$

$\leftarrow$ Favours OCS+antibiotics Favours OCS alone $\rightarrow$

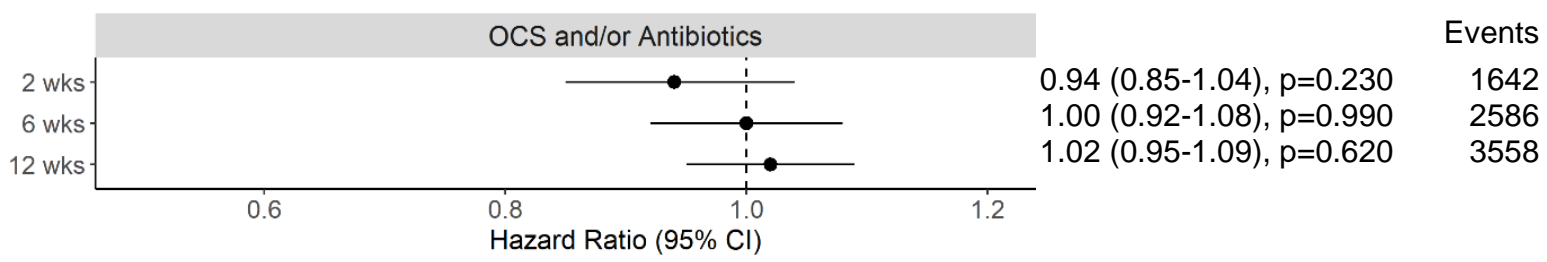

b) In 2-18 yr olds $(n=4,184)$

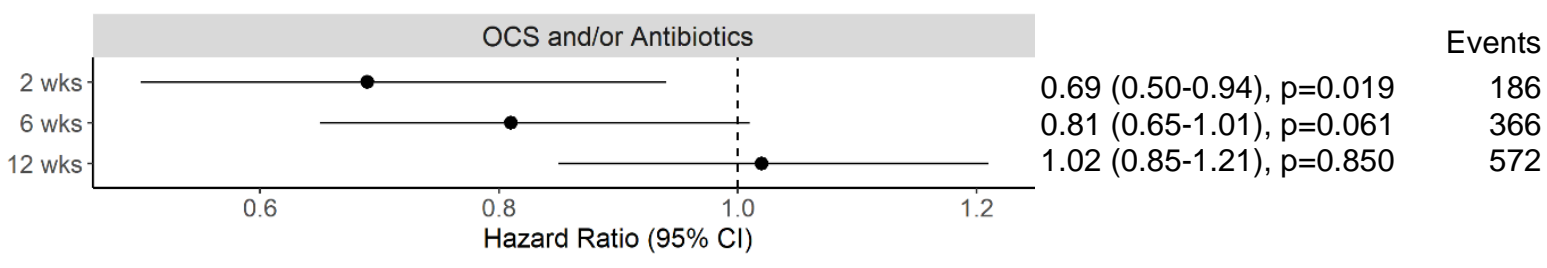


Supplementary figure 4 . Hazard ratios $(95 \% \mathrm{Cl})$ for oral corticosteroids (OCS) plus antibiotics compared to oral corticosteroids alone in adults with high (>0.2) and low (0-0.2) blood eosinophil count.

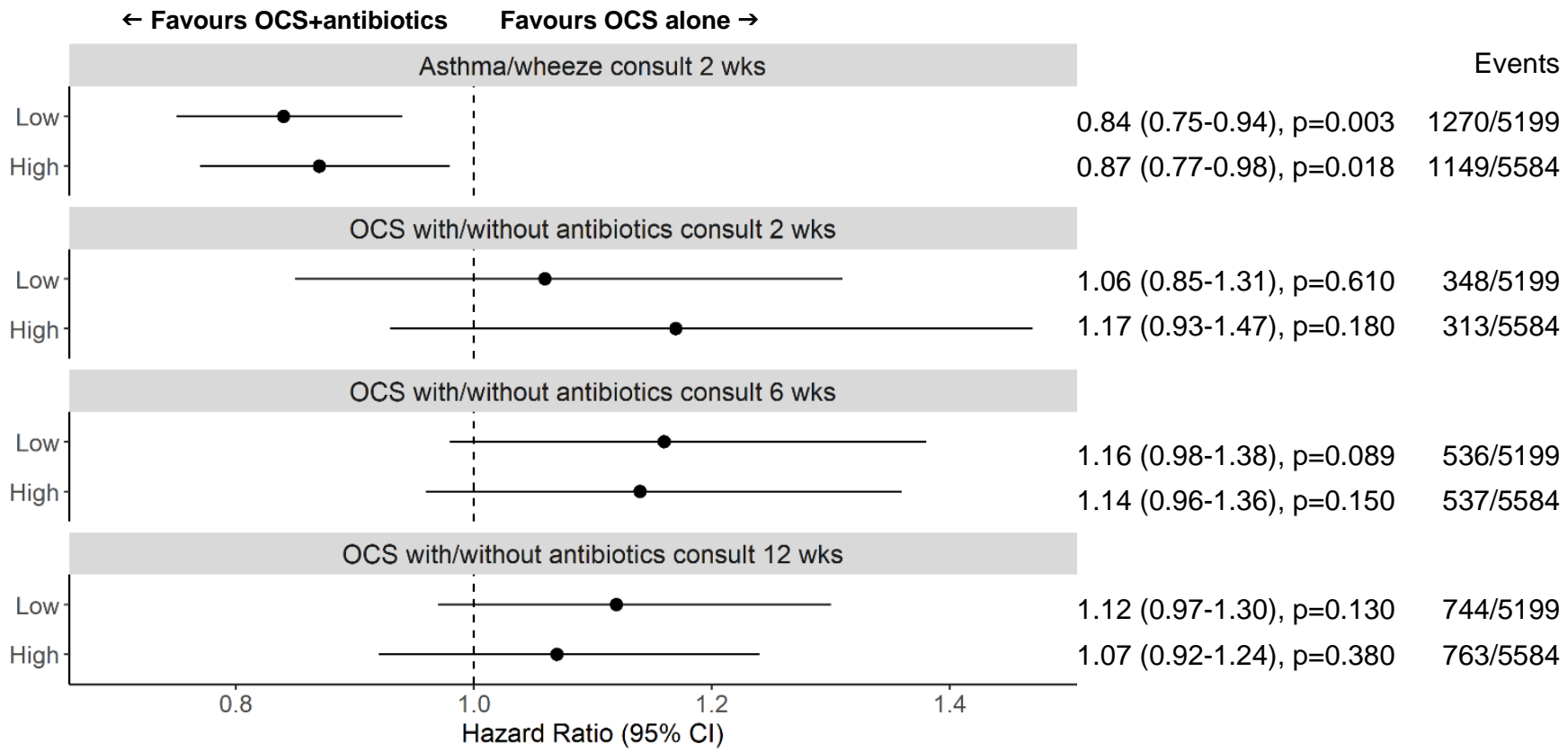




\section{Supplementary material}

Supplementary table 1. Demographic and clinical characteristics for 2-18 year olds. Values are $n(\%)$.

\begin{tabular}{|c|c|c|c|c|}
\hline & \multirow[b]{2}{*}{$\begin{array}{r}\text { Total } \\
(n=6,632)\end{array}$} & \multicolumn{3}{|c|}{ Treatment at Index Prescription Date } \\
\hline & & $\begin{array}{r}\text { OCS } \\
(n=4,538)\end{array}$ & $\begin{array}{r}\text { OCS + Antibiotic } \\
(\mathrm{n}=2,094)\end{array}$ & p-value \\
\hline \multicolumn{5}{|l|}{ Age, yrs } \\
\hline $2-5$ & $909(13.7 \%)$ & $624(13.8 \%)$ & $285(13.6 \%)$ & \multirow{3}{*}{$<0.001$} \\
\hline $6-12$ & $3,502(52.8 \%)$ & $2,468(54.4 \%)$ & $1,034(49.4 \%)$ & \\
\hline $13-18$ & $2,221(33.5 \%)$ & $1,446(31.9 \%)$ & $775(37.0 \%)$ & \\
\hline \multicolumn{5}{|l|}{ Sex } \\
\hline Female & $2,593(39.1 \%)$ & $1,780(39.2 \%)$ & $813(38.8 \%)$ & \multirow[t]{2}{*}{0.780} \\
\hline Male & $4,039(60.9 \%)$ & $2,758(60.8 \%)$ & $1,281(61.2 \%)$ & \\
\hline \multicolumn{5}{|c|}{ z-score Body Mass Index } \\
\hline Underweight & $233(4.6 \%)$ & $158(4.6 \%)$ & $75(4.5 \%)$ & \multirow[t]{5}{*}{0.011} \\
\hline Normal & $2,935(57.7 \%)$ & $1,985(57.9 \%)$ & $950(57.2 \%)$ & \\
\hline Overweight & $1,011(19.9 \%)$ & $664(19.4 \%)$ & $347(20.9 \%)$ & \\
\hline Obese & $909(17.9 \%)$ & $621(18.1 \%)$ & $288(17.3 \%)$ & \\
\hline Missing & $1,544(23.3 \%)$ & $1,110(24.5 \%)$ & $434(20.7 \%)$ & \\
\hline \multicolumn{5}{|l|}{ Smoking status } \\
\hline Current Smoker & $276(4.6 \%)$ & $141(3.4 \%)$ & $135(7.2 \%)$ & \multirow{3}{*}{$<0.001$} \\
\hline Ex-Smoker & $241(4.0 \%)$ & $175(4.3 \%)$ & $66(3.5 \%)$ & \\
\hline Non-Smoker & $5,456(91.3 \%)$ & $3,778(92.3 \%)$ & $1,678(89.3 \%)$ & \\
\hline Missing & $659(9.9 \%)$ & $444(9.8 \%)$ & $215(10.3 \%)$ & \\
\hline \multicolumn{5}{|c|}{ Global initiative for Asthma (GINA) category } \\
\hline Step 2 & $2,461(37.1 \%)$ & $1,660(36.6 \%)$ & $801(38.3 \%)$ & \multirow[t]{3}{*}{0.110} \\
\hline Step 3 & $2,634(39.7 \%)$ & $1,794(39.5 \%)$ & $840(40.1 \%)$ & \\
\hline Step 4 & $1,537(23.2 \%)$ & $1,084(23.9 \%)$ & $453(21.6 \%)$ & \\
\hline \multicolumn{5}{|c|}{ Eosinophil Count (x109/L) } \\
\hline$>0$ to 0.2 & $201(27.4 \%)$ & $129(26.6 \%)$ & $72(28.9 \%)$ & \multirow[t]{6}{*}{0.33} \\
\hline$>0.2$ to 0.4 & $191(26.0 \%)$ & $127(26.2 \%)$ & $64(25.7 \%)$ & \\
\hline$>0.4$ to 0.6 & $129(17.6 \%)$ & $90(18.6 \%)$ & $39(15.7 \%)$ & \\
\hline$>0.6$ to 0.8 & $88(12.0 \%)$ & $55(11.3 \%)$ & $33(13.3 \%)$ & \\
\hline$>0.8$ to 1 & $46(6.3 \%)$ & $36(7.4 \%)$ & $10(4.0 \%)$ & \\
\hline$>1$ & $79(10.8 \%)$ & $48(9.9 \%)$ & $31(12.4 \%)$ & \\
\hline Missing & $5,898(88.9 \%)$ & $4,053(89.3 \%)$ & $1,845(88.1 \%)$ & \\
\hline \multicolumn{5}{|c|}{ Season of Index Prescription Date } \\
\hline Autumn & $1,958(29.5 \%)$ & $1,298(28.6 \%)$ & $660(31.5 \%)$ & \multirow[t]{4}{*}{$<0.001$} \\
\hline Winter & $1,932(29.1 \%)$ & $1,257(27.7 \%)$ & $675(32.2 \%)$ & \\
\hline Spring & $1,438(21.7 \%)$ & $1,017(22.4 \%)$ & $421(20.1 \%)$ & \\
\hline Summer & $1,304(19.7 \%)$ & $966(21.3 \%)$ & $338(16.1 \%)$ & \\
\hline \multicolumn{5}{|c|}{ Year of Index Prescription Date } \\
\hline $2004-2006$ & $2,328(35.1 \%)$ & $1,668(36.8 \%)$ & $660(31.5 \%)$ & $<0.001$ \\
\hline $2007-2009$ & $2,178(32.8 \%)$ & $1,486(32.8 \%)$ & $692(33.1 \%)$ & \\
\hline 2010-2012 & $1,556(23.5 \%)$ & $1,004(22.1 \%)$ & $552(26.4 \%)$ & \\
\hline 2013-2014 & $570(8.6 \%)$ & $380(8.4 \%)$ & $190(9.1 \%)$ & \\
\hline $\begin{array}{l}\text { Number of asthm } \\
\text { total }\end{array}$ & onsults in basel & 6 months & & \\
\hline 0 & $2,437(36.8 \%)$ & $1,647(36.3 \%)$ & $790(37.7 \%)$ & 0.460 \\
\hline $1-5$ & $4,079(61.5 \%)$ & $2,813(62.0 \%)$ & $1,266(60.5 \%)$ & \\
\hline $6-10$ & $107(1.6 \%)$ & $73(1.6 \%)$ & $34(1.6 \%)$ & \\
\hline $11-15$ & $8(0.1 \%)$ & $5(0.1 \%)$ & $3(0.1 \%)$ & \\
\hline $16-20$ & $1(0.0 \%)$ & $0(0.0 \%)$ & $1(0.1 \%)$ & \\
\hline with Short-Actin & ist (SABA) pres & ption & & \\
\hline 0 & $2,437(36.8 \%)$ & $1,647(36.3 \%)$ & $790(37.7 \%)$ & 0.110 \\
\hline 1 & $3,229(48.7 \%)$ & $2,203(48.6 \%)$ & $1,026(49.0 \%)$ & \\
\hline 2 & $966(14.6 \%)$ & $688(15.2 \%)$ & $278(13.3 \%)$ & \\
\hline with antibiotic $p$ & & & & \\
\hline 0 & $6,024(90.8 \%)$ & $4,144(91.3 \%)$ & $1,880(89.8 \%)$ & 0.074 \\
\hline 1 & $562(8.5 \%)$ & $368(8.1 \%)$ & $194(9.3 \%)$ & \\
\hline 2 & $44(0.7 \%)$ & $24(0.5 \%)$ & $20(1.0 \%)$ & \\
\hline 3 & $1(0.0 \%)$ & $1(0.0 \%)$ & $0(0.0 \%)$ & \\
\hline 4 & $1(0.0 \%)$ & $1(0.0 \%)$ & $0(0.0 \%)$ & \\
\hline
\end{tabular}

OCS: Oral Corticosteroids. Percentages are given as non-missing. 
Supplementary table 2. Demographic and clinical characteristics for 19-65 year olds.

Values are $n(\%)$.

\begin{tabular}{|c|c|c|c|c|}
\hline & \multirow[b]{2}{*}{$\begin{array}{r}\text { Total } \\
(n=22,005)\end{array}$} & \multicolumn{3}{|c|}{ Treatment at Index Prescription Date } \\
\hline & & $\begin{array}{r}\text { OCS } \\
(n=11,993)\end{array}$ & $\begin{array}{r}\text { OCS + Antibiotic } \\
(n=10,012)\end{array}$ & p-value \\
\hline \multicolumn{5}{|l|}{ Age, yrs } \\
\hline $19-25$ & $1,956(8.9 \%)$ & $1,176(9.8 \%)$ & $780(7.8 \%)$ & $<0.001$ \\
\hline $26-35$ & $3,904(17.7 \%)$ & $2,288(19.1 \%)$ & $1,616(16.1 \%)$ & \\
\hline $36-45$ & $5,723(26.0 \%)$ & $3,224(26.9 \%)$ & $2,499(25.0 \%)$ & \\
\hline $46-55$ & $5,417(24.6 \%)$ & $2,830(23.6 \%)$ & $2,587(25.8 \%)$ & \\
\hline $56-65$ & $5.005(22.7 \%)$ & $2,475(20.6 \%)$ & $2,530(25.3 \%)$ & \\
\hline \multicolumn{5}{|l|}{ Sex } \\
\hline Female & $14,407(65.5 \%)$ & $7,958(66.4 \%)$ & $6,449(64.4 \%)$ & 0.003 \\
\hline Male & $7,598(34.5 \%)$ & $4,035(33.6 \%)$ & $3,563(35.6 \%)$ & \\
\hline \multicolumn{5}{|l|}{ Body Mass Index } \\
\hline Underweight & $381(1.8 \%)$ & $216(1.8 \%)$ & $165(1.7 \%)$ & 0.004 \\
\hline Normal & $5,810(27.0 \%)$ & $3,274(27.9 \%)$ & $2,536(25.9 \%)$ & \\
\hline Overweight & $6,910(32.1 \%)$ & $3,757(32.1 \%)$ & $3,153(32.2 \%)$ & \\
\hline Obese & $8,417(39.1 \%)$ & $4,474(38.2 \%)$ & $3,943(40.2 \%)$ & \\
\hline Missing & $487(2.2 \%)$ & $272(2.3 \%)$ & $215(2.1 \%)$ & \\
\hline \multicolumn{5}{|l|}{ Smoking status } \\
\hline Current Smoker & $4,854(22.4 \%)$ & 2,335 (19.9\%) & $2,519(25.6 \%)$ & $<0.001$ \\
\hline Ex-Smoker & $5,782(26.8 \%)$ & $3,132(26.6 \%)$ & $2,650(26.9 \%)$ & \\
\hline Non-Smoker & $10,978(50.8 \%)$ & $6,291(53.5 \%)$ & $4,687(47.6 \%)$ & \\
\hline Missing & $391(1.8 \%)$ & $235(2.0 \%)$ & $156(1.6 \%)$ & \\
\hline \multicolumn{5}{|c|}{ Global initiative for Asthma (GINA) category } \\
\hline Step 2 & $6,471(29.4 \%)$ & $3,517(29.3 \%)$ & $2,954(29.5 \%)$ & 0.960 \\
\hline Step 3 & $6,104(27.7 \%)$ & $3,329(27.8 \%)$ & $2,775(27.7 \%)$ & \\
\hline Step 4 & $9,430(42.9 \%)$ & $5,147(42.9 \%)$ & $4,283(42.8 \%)$ & \\
\hline \multicolumn{5}{|c|}{ Eosinophil Count (x109/L) } \\
\hline$>0$ to 0.2 & $5,658(48.2 \%)$ & $3,066(48.5 \%)$ & $2,592(47.9 \%)$ & 0.090 \\
\hline$>0.2$ to 0.4 & $3,940(33.6 \%)$ & $2,099(33.2 \%)$ & $1,841(34.0 \%)$ & \\
\hline$>0.4$ to 0.6 & $1,390(11.8 \%)$ & $725(11.5 \%)$ & $665(12.3 \%)$ & \\
\hline$>0.6$ to 0.8 & $450(3.8 \%)$ & $270(4.3 \%)$ & $180(3.3 \%)$ & \\
\hline$>0.8$ to 1 & $164(1.4 \%)$ & $91(1.4 \%)$ & $73(1.3 \%)$ & \\
\hline$>1$ & $134(1.1 \%)$ & $74(1.2 \%)$ & $60(1.1 \%)$ & \\
\hline Missing & $10,269(46.7 \%)$ & $5,668(47.3 \%)$ & $4,601(46.0 \%)$ & \\
\hline \multicolumn{5}{|c|}{ Season of Index Prescription Date } \\
\hline Autumn & $5,823(26.5 \%)$ & $3,178(26.5 \%)$ & $2,645(26.4 \%)$ & $<0.001$ \\
\hline Winter & $6,981(31.7 \%)$ & $3,474(29.0 \%)$ & $3,507(35.0 \%)$ & \\
\hline Spring & $4,806(21.8 \%)$ & $2,661(22.2 \%)$ & $2,145(21.4 \%)$ & \\
\hline Summer & $4,395(20.0 \%)$ & $2,680(22.4 \%)$ & $1,715(17.1 \%)$ & \\
\hline \multicolumn{5}{|c|}{ Year of Index Prescription Date } \\
\hline $2004-2006$ & $6,780(30.8 \%)$ & $4,050(33.8 \%)$ & $2,730(27.3 \%)$ & $<0.001$ \\
\hline $2007-2009$ & $7,169(32.6 \%)$ & $3,970(33.1 \%)$ & $3,199(32.0 \%)$ & \\
\hline $2010-2012$ & $5,586(25.4 \%)$ & $2,812(23.5 \%)$ & $2,774(27.7 \%)$ & \\
\hline $2013-2014$ & $2,470(11.2 \%)$ & $1,161(9.7 \%)$ & $1,309(13.1 \%)$ & \\
\hline \multicolumn{5}{|c|}{$\begin{array}{l}\text { Number of asthma/wheeze consults in baseline } 6 \text { months } \\
\text { total }\end{array}$} \\
\hline 0 & $10,304(46.8 \%)$ & $5,483(45.7 \%)$ & $4,821(48.2 \%)$ & 0.003 \\
\hline $1-5$ & $11,351(51.6 \%)$ & $6,324(52.7 \%)$ & $5,027(50.2 \%)$ & \\
\hline $6-10$ & $306(1.4 \%)$ & $162(1.4 \%)$ & $144(1.4 \%)$ & \\
\hline $11-15$ & $38(0.2 \%)$ & $19(0.2 \%)$ & $19(0.2 \%)$ & \\
\hline $16-20$ & $5(0.0 \%)$ & $4(0.0 \%)$ & $1(0.0 \%)$ & \\
\hline $26-30$ & $1(0.0 \%)$ & $1(0.0 \%)$ & $0(0.0 \%)$ & \\
\hline \multicolumn{5}{|c|}{ with Short-Acting Beta Agonist (SABA) prescription } \\
\hline 0 & $10,304(46.8 \%)$ & $5,483(45.7 \%)$ & $4,821(48.2 \%)$ & $<0.001$ \\
\hline 1 & $9,654(43.9 \%)$ & $5,332(44.5 \%)$ & $4,322(43.2 \%)$ & \\
\hline 2 & $2,047(9.3 \%)$ & $1,178(9.8 \%)$ & $869(8.7 \%)$ & \\
\hline \multicolumn{5}{|c|}{ with antibiotic prescription } \\
\hline 0 & $20,114(91.4 \%)$ & $10,909(91.0 \%)$ & $9,205(91.9 \%)$ & 0.098 \\
\hline 1 & $1,713(7.8 \%)$ & $983(8.2 \%)$ & $730(7.3 \%)$ & \\
\hline 2 & $150(0.7 \%)$ & $84(0.7 \%)$ & $66(0.7 \%)$ & \\
\hline 3 & $23(0.1 \%)$ & $13(0.1 \%)$ & $10(0.1 \%)$ & \\
\hline 4 & $5(0.0 \%)$ & $4(0.0 \%)$ & $1(0.0 \%)$ & \\
\hline
\end{tabular}

OCS: Oral Corticosteroids. Percentages are given as non-missing. 
Supplementary table 3. Comorbidities for 2-18 year olds and 19-65 year olds

\begin{tabular}{|c|c|c|c|c|}
\hline & \multicolumn{4}{|c|}{$19-65$ year olds } \\
\hline & $\begin{array}{r}\text { Total } \\
(n=22,005)\end{array}$ & $\begin{array}{r}\text { OCS } \\
(n=11,993)\end{array}$ & $\begin{array}{r}\text { OCS + } \\
\text { Antibiotic } \\
(n=10,012)\end{array}$ & p-value \\
\hline$\overline{\text { COPD }}$ & $0(0.0 \%)$ & $0(0.0 \%)$ & $0(0.0 \%)$ & $\overline{1.000}$ \\
\hline Active rhinitis & $3,218(14.6 \%)$ & $1,885(15.7 \%)$ & $1,333(13.3 \%)$ & $<0.001$ \\
\hline Active GERD & $1,071(4.9 \%)$ & $544(4.5 \%)$ & $527(5.3 \%)$ & 0.013 \\
\hline Active eczema & $927(4.2 \%)$ & $536(4.5 \%)$ & $391(3.9 \%)$ & 0.040 \\
\hline Osteoporosis & $246(1.1 \%)$ & $127(1.1 \%)$ & $119(1.2 \%)$ & 0.370 \\
\hline Chronic Kidney Disease & $226(1.0 \%)$ & $114(1.0 \%)$ & $112(1.1 \%)$ & 0.230 \\
\hline Diabetes & $940(4.3 \%)$ & $478(4.0 \%)$ & $462(4.6 \%)$ & 0.023 \\
\hline Hypertension & $1124(5.1 \%)$ & $584(4.9 \%)$ & $540(5.4 \%)$ & 0.080 \\
\hline Ischaemic Heart Disease & $625(2.8 \%)$ & $311(2.6 \%)$ & $314(3.1 \%)$ & 0.016 \\
\hline Cardiovascular Disease & $1,437(6.5 \%)$ & $728(6.1 \%)$ & $709(7.1 \%)$ & 0.003 \\
\hline Heart Failure & $50(0.2 \%)$ & $23(0.2 \%)$ & $27(0.3 \%)$ & 0.260 \\
\hline Myocardial Infarction & $184(0.8 \%)$ & $92(0.8 \%)$ & $92(0.9 \%)$ & 0.230 \\
\hline Cerebrovascular Disease & $271(1.2 \%)$ & $126(1.1 \%)$ & $145(1.4 \%)$ & 0.008 \\
\hline \multirow[t]{3}{*}{ Anxiety and/or Depression } & $1,887(8.6 \%)$ & $1,038(8.7 \%)$ & $849(8.5 \%)$ & 0.650 \\
\hline & \multicolumn{4}{|c|}{ 2-18 year olds } \\
\hline & $\begin{array}{r}\text { Total } \\
(n=6,632)\end{array}$ & $\begin{array}{r}\text { OCS } \\
(n=4,538)\end{array}$ & $\begin{array}{r}\text { OCS + } \\
\text { Antibiotic } \\
(n=2,094)\end{array}$ & p-value \\
\hline Active rhinitis & $909(13.7 \%)$ & $624(13.8 \%)$ & $285(13.6 \%)$ & 0.910 \\
\hline Active eczema & $599(9.0 \%)$ & $406(8.9 \%)$ & $193(9.2 \%)$ & 0.710 \\
\hline
\end{tabular}


Supplementary table 4. Comorbidities for 2-18 year olds and 19-65 year olds, following propensity score matching

\begin{tabular}{|c|c|c|c|c|}
\hline & \multicolumn{4}{|c|}{$19-65$ year olds } \\
\hline & $\begin{array}{r}\text { Total } \\
(n=20,024)\end{array}$ & $\begin{array}{r}\text { OCS } \\
(n=10,012)\end{array}$ & $\begin{array}{r}\text { OCS + } \\
\text { Antibiotic } \\
(n=10,012)\end{array}$ & p-value \\
\hline$\overline{\text { COPD }}$ & $0(0.0 \%)$ & $0(0.0 \%)$ & $0(0.0 \%)$ & 1.0 \\
\hline Active rhinitis & $2,879(14.4 \%)$ & $1,546(15.4 \%)$ & $1,333(13.3 \%)$ & $<0.001$ \\
\hline Active GERD & $1,015(5.1 \%)$ & $488(4.9 \%)$ & $527(5.3 \%)$ & 0.22 \\
\hline Active eczema & $829(4.1 \%)$ & $438(4.4 \%)$ & $391(3.9 \%)$ & 0.10 \\
\hline Osteoporosis & $231(1.2 \%)$ & $112(1.1 \%)$ & $119(1.2 \%)$ & 0.69 \\
\hline Chronic Kidney Disease & $213(1.1 \%)$ & $101(1.0 \%)$ & $112(1.1 \%)$ & 0.49 \\
\hline Diabetes & $904(4.5 \%)$ & $442(4.4 \%)$ & $462(4.6 \%)$ & 0.52 \\
\hline Hypertension & $1,077(5.4 \%)$ & $537(5.4 \%)$ & $540(5.4 \%)$ & 0.95 \\
\hline Ischaemic Heart Disease & $597(3.0 \%)$ & $283(2.8 \%)$ & $314(3.1 \%)$ & 0.21 \\
\hline Cardiovascular Disease & $1,365(6.8 \%)$ & $656(6.6 \%)$ & $709(7.1 \%)$ & 0.14 \\
\hline Heart Failure & $49(0.2 \%)$ & $22(0.2 \%)$ & $27(0.3 \%)$ & 0.57 \\
\hline Myocardial Infarction & $179(0.9 \%)$ & $87(0.9 \%)$ & $92(0.9 \%)$ & 0.76 \\
\hline Cerebrovascular Disease & $259(1.3 \%)$ & $114(1.1 \%)$ & $145(1.4 \%)$ & 0.06 \\
\hline \multirow[t]{3}{*}{ Anxiety and/or Depression } & $1,717(8.6 \%)$ & $868(8.7 \%)$ & $849(8.5 \%)$ & 0.65 \\
\hline & \multicolumn{4}{|c|}{ 2-18 year olds } \\
\hline & $\begin{array}{r}\text { Total } \\
(\mathrm{n}=4,184) \\
\end{array}$ & $\begin{array}{r}\text { OCS } \\
(n=2,092) \\
\end{array}$ & $\begin{array}{r}\text { OCS + } \\
\text { Antibiotic } \\
(n=2,092) \\
\end{array}$ & p-value \\
\hline Active rhinitis & $561(13.4 \%)$ & $277(13.2 \%)$ & $284(13.6 \%)$ & 0.79 \\
\hline Active eczema & 367 (8.8\%) & 175 (8.4\%) & $192(9.2 \%)$ & 0.38 \\
\hline
\end{tabular}


STROBE 2007 (v4) Statement-Checklist of items that should be included in reports of cohort studies

\begin{tabular}{|c|c|c|c|}
\hline Section/Topic & $\begin{array}{l}\text { Item } \\
\#\end{array}$ & Recommendation & Reported on page \# \\
\hline \multirow[t]{2}{*}{ Title and abstract } & \multirow[t]{2}{*}{1} & (a) Indicate the study's design with a commonly used term in the title or the abstract & $1-2$ \\
\hline & & (b) Provide in the abstract an informative and balanced summary of what was done and what was found & $1-2$ \\
\hline \multicolumn{4}{|l|}{ Introduction } \\
\hline Background/rationale & 2 & Explain the scientific background and rationale for the investigation being reported & 3 \\
\hline Objectives & 3 & State specific objectives, including any prespecified hypotheses & 3 \\
\hline \multicolumn{4}{|l|}{ Methods } \\
\hline Study design & 4 & Present key elements of study design early in the paper & $3-4$ \\
\hline Setting & 5 & $\begin{array}{l}\text { Describe the setting, locations, and relevant dates, including periods of recruitment, exposure, follow-up, and data } \\
\text { collection }\end{array}$ & 3-4 \\
\hline \multirow[t]{2}{*}{ Participants } & \multirow[t]{2}{*}{6} & (a) Give the eligibility criteria, and the sources and methods of selection of participants. Describe methods of follow-up & 4 \\
\hline & & (b) For matched studies, give matching criteria and number of exposed and unexposed & 5 \\
\hline Variables & 7 & $\begin{array}{l}\text { Clearly define all outcomes, exposures, predictors, potential confounders, and effect modifiers. Give diagnostic criteria, if } \\
\text { applicable }\end{array}$ & $4-5$ \\
\hline $\begin{array}{l}\text { Data sources/ } \\
\text { measurement }\end{array}$ & $8^{*}$ & $\begin{array}{l}\text { For each variable of interest, give sources of data and details of methods of assessment (measurement). Describe } \\
\text { comparability of assessment methods if there is more than one group }\end{array}$ & $3-4$ \\
\hline Bias & 9 & Describe any efforts to address potential sources of bias & 5 \\
\hline Study size & 10 & Explain how the study size was arrived at & 5,12 \\
\hline Quantitative variables & 11 & $\begin{array}{l}\text { Explain how quantitative variables were handled in the analyses. If applicable, describe which groupings were chosen and } \\
\text { why }\end{array}$ & $4-5$ \\
\hline \multirow[t]{5}{*}{ Statistical methods } & \multirow[t]{5}{*}{12} & (a) Describe all statistical methods, including those used to control for confounding & $4-5$ \\
\hline & & (b) Describe any methods used to examine subgroups and interactions & 5 \\
\hline & & (c) Explain how missing data were addressed & $4-5$ \\
\hline & & (d) If applicable, explain how loss to follow-up was addressed & 4 \\
\hline & & (e) Describe any sensitivity analyses & NA \\
\hline \multicolumn{3}{|l|}{ Results } & \\
\hline
\end{tabular}




\begin{tabular}{|c|c|c|c|}
\hline Participants & $13^{*}$ & $\begin{array}{l}\text { (a) Report numbers of individuals at each stage of study-eg numbers potentially eligible, examined for eligibility, confirmed } \\
\text { eligible, included in the study, completing follow-up, and analysed }\end{array}$ & 12 \\
\hline & & (b) Give reasons for non-participation at each stage & 12 \\
\hline & & (c) Consider use of a flow diagram & 12 \\
\hline \multirow[t]{3}{*}{ Descriptive data } & $14^{*}$ & $\begin{array}{l}\text { (a) Give characteristics of study participants (eg demographic, clinical, social) and information on exposures and potential } \\
\text { confounders }\end{array}$ & $13-15$ \\
\hline & & (b) Indicate number of participants with missing data for each variable of interest & 13-15 \\
\hline & & (c) Summarise follow-up time (eg, average and total amount) & 4 \\
\hline Outcome data & $15^{*}$ & Report numbers of outcome events or summary measures over time & 18 \\
\hline \multirow[t]{3}{*}{ Main results } & 16 & $\begin{array}{l}\text { (a) Give unadjusted estimates and, if applicable, confounder-adjusted estimates and their precision (eg, } 95 \% \text { confidence } \\
\text { interval). Make clear which confounders were adjusted for and why they were included }\end{array}$ & 16,17 \\
\hline & & (b) Report category boundaries when continuous variables were categorized & 16 \\
\hline & & (c) If relevant, consider translating estimates of relative risk into absolute risk for a meaningful time period & $5-6,18$ \\
\hline Other analyses & 17 & Report other analyses done-eg analyses of subgroups and interactions, and sensitivity analyses & 6 \\
\hline \multicolumn{4}{|l|}{ Discussion } \\
\hline Key results & 18 & Summarise key results with reference to study objectives & $6-7$ \\
\hline \multicolumn{4}{|l|}{ Limitations } \\
\hline Interpretation & 20 & $\begin{array}{l}\text { Give a cautious overall interpretation of results considering objectives, limitations, multiplicity of analyses, results from } \\
\text { similar studies, and other relevant evidence }\end{array}$ & $6-8$ \\
\hline Generalisability & 21 & Discuss the generalisability (external validity) of the study results & $7-8$ \\
\hline \multicolumn{4}{|l|}{ Other information } \\
\hline Funding & 22 & $\begin{array}{l}\text { Give the source of funding and the role of the funders for the present study and, if applicable, for the original study on } \\
\text { which the present article is based }\end{array}$ & 9 \\
\hline
\end{tabular}

*Give information separately for cases and controls in case-control studies and, if applicable, for exposed and unexposed groups in cohort and cross-sectional studies.

Note: An Explanation and Elaboration article discusses each checklist item and gives methodological background and published examples of transparent reporting. The STROBE checklist is best used in conjunction with this article (freely available on the Web sites of PLoS Medicine at http://www.plosmedicine.org/, Annals of Internal Medicine at http://www.annals.org/, and Epidemiology at http://www.epidem.com/). Information on the STROBE Initiative is available at www.strobe-statement.org. 